\title{
ON THE ACOUSITC RESPONSE OF ULTRASOUND AND \\ INDUCED CELL DEATH
}

\author{
By \\ Fatimah Alsaiari \\ B.Sc. King Faisal University, 2008 \\ Dammam, Saudi Arabia \\ A thesis \\ presented to Ryerson University \\ In partial fulfillment of \\ the requirements for the degree of \\ Master of Science \\ In the Program of \\ Biomedical Physics
}

Toronto, Ontario, Canada, 2014

(C) Fatimah Alsaiari, 2014 


\section{Author's Declaration}

I hereby declare that I am the sole author of this thesis or dissertation. This is a true copy of the thesis, including any required final revisions as accepted by my examiners.

I authorize Ryerson University to lend this thesis or dissertation to other institutions or individuals for the purpose of scholarly research.

I further authorize Ryerson University to reproduce this thesis or dissertation by photocopying or by other means, in total or in part, at the request of other institutions or individuals for the purpose of scholarly research.

I understand that my thesis may be made electronically available to the public.

Fatimah Alsaiari 


\begin{abstract}
On the acoustic response of ultrasound and microbubble induced cell death
\end{abstract}

\author{
Fatimah Alsaiari \\ Master of Science, Biomedical Physics \\ Ryerson University, 2014.
}

Ultrasonically-stimulated microbubbles can enhance cell membrane permeability and decrease cell viability where the underlying acoustic mechanism has been associated with both non-inertial and inertial cavitation. In this study, breast cancer cells (MDA-MB-231) were exposed to $0.5 \mathrm{MHz}$ ultrasound pulses of $16 \mu \mathrm{s}$ duration at varying peak negative pressures (PNP: $218 \mathrm{kPa}$, $335 \mathrm{kPa}$ and $908 \mathrm{kPa}$ ) and pulse repetition period (PRP 10ms and 100ms) in the presence of Definity microbubbles $(3.3 \% \mathrm{v} / \mathrm{v})$. The acoustic response of microbubbles was measured using passive cavitation detection with $2.25 \mathrm{MHz}$ transducer, and characterized by their frequency a cavitation dose (CD). Results show that the number of non-viable cells and integrated cavitation dose (ICD) significantly increases with PNP, whereas no significant differences were found between 10ms and 100ms PRPs. In this study, no correlation was found between (ICD) and cell non-viability. 


\section{Acknowledgments}

There are many people I would like to acknowledge that contributed to the success of this paper and the completion of my Master's degree.

First, I would like to express the deepest appreciation to my supervisor Prof. Raffi Karshafian for giving me the opportunity to study, research, and pursue my master's degree at Ryerson University. Without his guidance and positive attitude, this milestone in my life would have not been possible. I began this journey with very little knowledge of the research field when I first started in Prof. Karshafian's lab. I can honestly say that I have gained a true passion for science. Thank you for taking me under your wing, your guidance had made me an even more confident and curious scientist.

Second, I would like to thank my supervisor committee members Drs. Jahan Tavakkoli and Alexandre Douplik who gave me a great experience and provided insightful thoughts and discussion. I valued their advice and insight for every step of my progress.

Third, I would like to thank my King Abdullah who gave me a chance to continue my education in Canada and complete my Master's degree. I would like also to thank the Saudi cultural bureau for their continued support in Canada.

Furthermore, I would like to thank my lab group members especially Narbeh Edjiu, Farah Hussein, Shadab Momin, Amin Jafari and Anna Sorensen for supporting my study.

Finally, I would like to thank my family who were always there to cheer me up and stood by me through the good and bad times. 


\section{Dedication}

To my husband, Rahed Alshamasy, and our children

To my mother, brother and sisters, especially "Sharifah Alsaiari" 
$\underline{\text { Table of Contents }}$

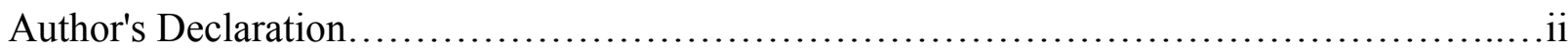

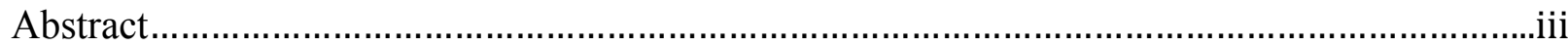

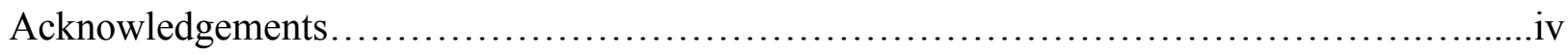

Dedication........................................................................

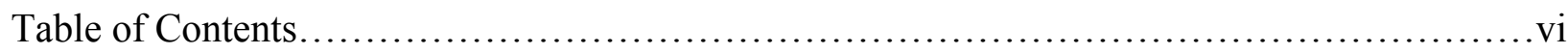

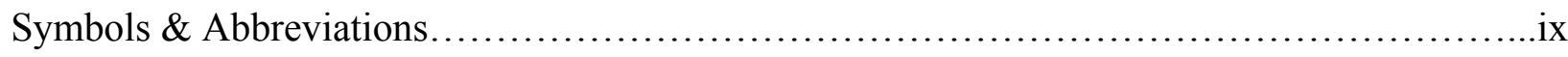

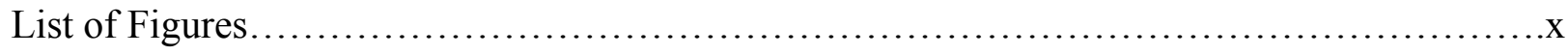

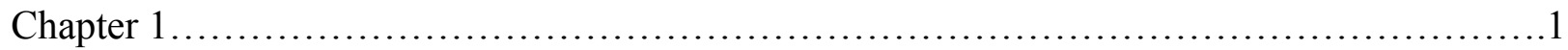

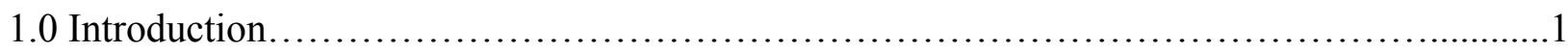

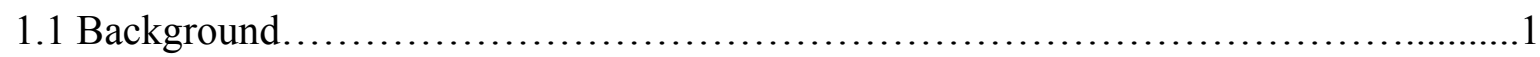

1.2 Ultrasound Physics......................................................

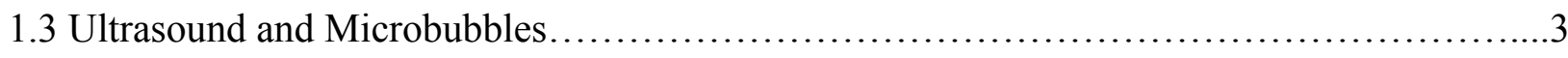

1.3.1 Microbubble Contrast Agents...........................................

1.3.2 Microbubble Physics...................................................

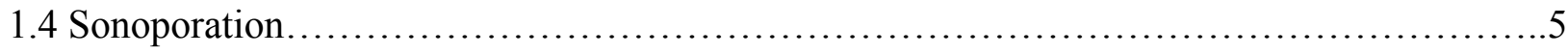

1.5 Microbubble Acoustic Response . ............................................ 8

1.5.1 Microbubble Characterization.................................................

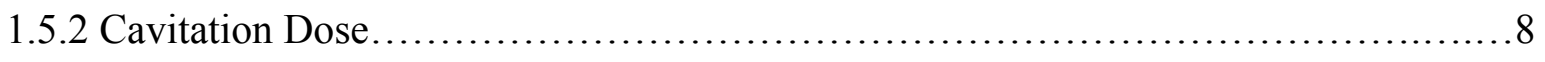

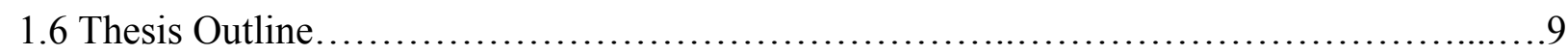




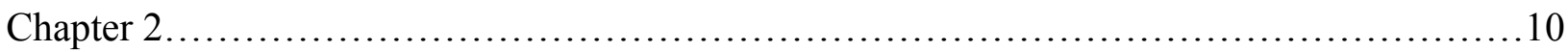

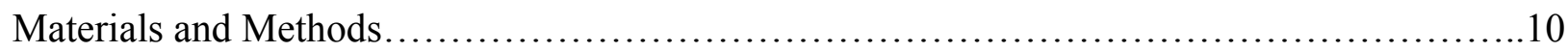

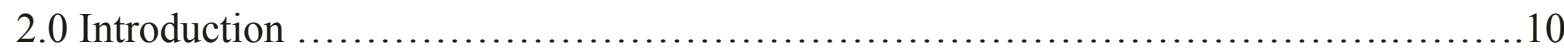

2.1In vitro Cell model............................................................... 10

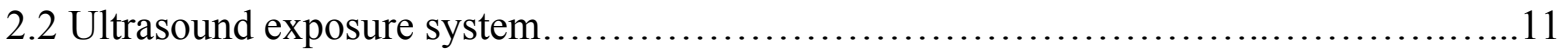

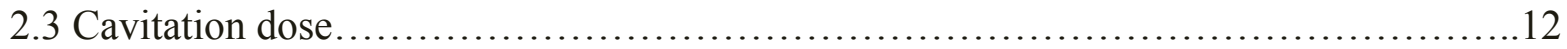

2.4 Microbubble conrast agent.......................................................

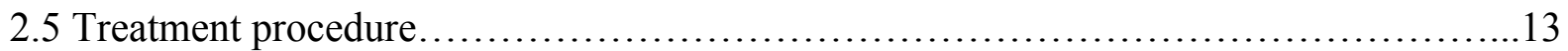

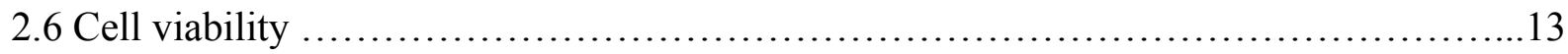

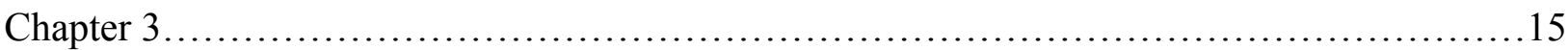

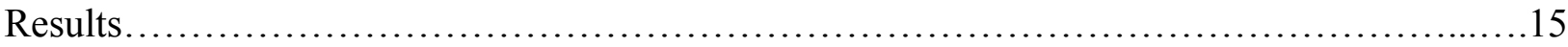

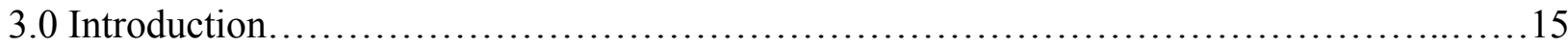

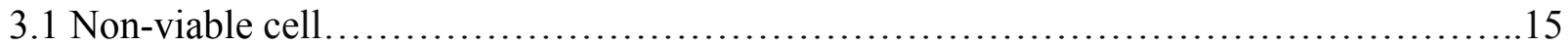

3.2 Acoustic characteristics of Microbubbles.............................................. 16

3.2.1 Effect of peak negative pressure.............................................. 17

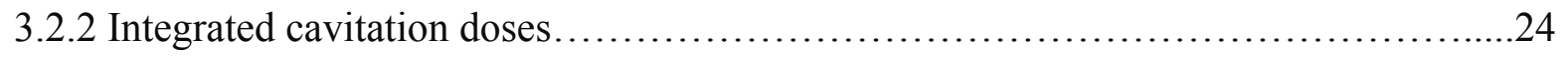

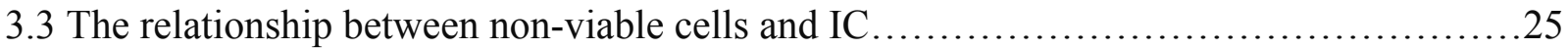

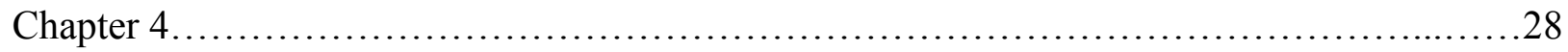




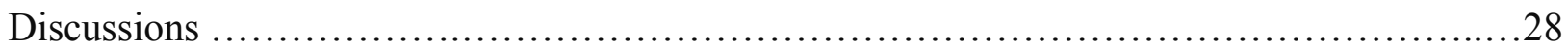

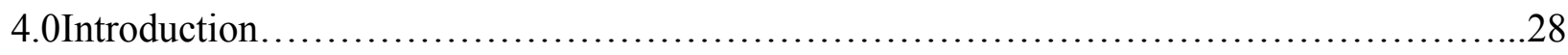

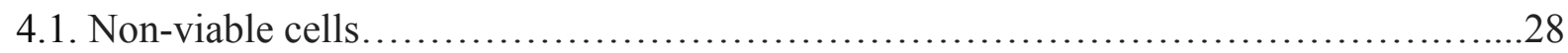

4.2 Acoustic characteristics of Microbubbles.......................................... 28

4.3 Relationship of non-viable cells and integrated cavitation dose ........................30

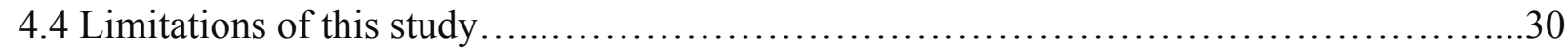

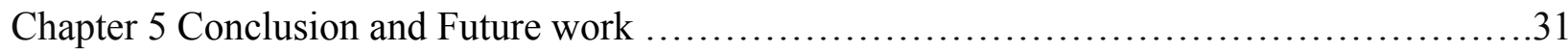

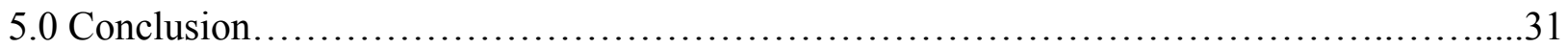

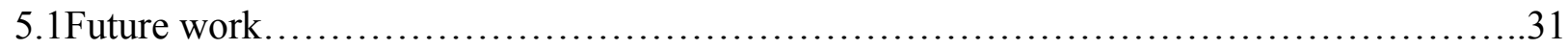

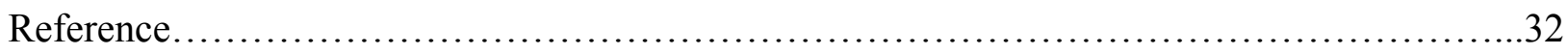




\section{LIST OF SYMBOLS AND ABBREVIATIONS}

\begin{tabular}{ll} 
PNP & Peak negative pressure \\
PCF & Pulse center frequency \\
CD & Cavitation dose \\
US & Ultrasound \\
MB & Microbubbles \\
RMS & Root mean square \\
RA & Receiver amplifier \\
TD & Time domain signal \\
ICD & Integrated cavitation dose \\
UCAs & Ultrasound contrast agents \\
PRP & Pulse Repetition Period \\
USMB & Ultrasound and Microbubbles \\
HR & Harmonic \\
\hline &
\end{tabular}




\section{List of Figures}

Figure 1.1: The acoustic emission spectrum for bubbles using the passive cavitation detection measurement. The figure includes fundamental signals, 2nd Harmonic, Ultra-Harmonic, 2nd

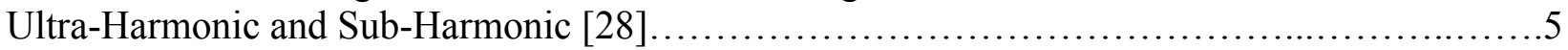

Figure 1.2: Schematic diagram of ultrasound and microbubble. Mirobubble on US filed

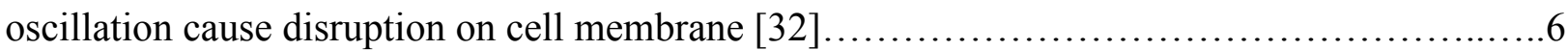

Figure 1.3: The acoustical mechanisms associated with ultrasonically-stimulated microbubbles include microstreaming induced by oscillating microbubbles, shock waves due to non-stable cavitation, and micro-jet formation due to collapsing microbubbles. A: drug molecules; B: stable cavitation of microbubbles; C: Microstreaming; D: non stable cavitation of microbubbles produce shock waves; E: fluid microjet; F: cell membrane disruption; G: non ruptured cell membrane with increase permeability due to USMB; $\mathrm{H}$ : cell membrane damage due to shock waves and microstreaming; I: extravascular tissue; J: microbubble loaded with drug molecules on its surface; K: microbubbles loaded with drug molecules in lipophilic phase; L: micelle loaded with drug molecule lipophilic phase; M: liposome with aqueous interior; N: targeted microbubble [20]

Figure 2.1: The schematic diagram of the ultrasound exposure system. Microbubbles and cells were exposed in a cylindrical chamber with a internal diameter of $8 \mathrm{~mm}$ and a height of $90 \mathrm{~mm}$; $30 \mathrm{~mm}$ was covered with acoustic membrane. Microbubbles were exposed to an ultrasound field using a $0.5 \mathrm{MHz}$ transducer and emissions were received using $2.25 \mathrm{MHz}$ transducer. Harmonic and ultr-harmonic frequencies were detected. 14

Figure 3.1: Non-viable cell of MDA-MB-231 cells exposed to a $0.5 \mathrm{MHz}$ center frequency at varying acoustic pressures $(218 \mathrm{kPa}, 335 \mathrm{kPa}, 908 \mathrm{kPa})$ with a $10 \mathrm{~ms}$ (black bars) and $100 \mathrm{~ms}$ (grey bars) PRP and total treatment time of $30 \mathrm{~s}$ in $3.3 \%$ Definity volume concentration. The percentages of Non-viable cells are shown with respect to different pressures at center

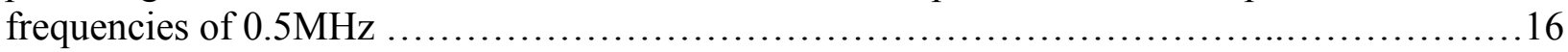

Figure 3.2:The acoustic behavior of Definity microbubbles at varying acoustic pressure at $10 \mathrm{~ms}$ PRP. The first column represents Time Domain Signal of the echo received, where the x-axis represents the time y-axis represents the amplitude. The second column represents the corresponding FFT of the time domain signal, where x-axis represents the frequency of the waves emitted from the microbubble and the y-axis represents the amplitude of the wave 18

Figure 3.3:The acoustic behaviour of Definity microbubbles at varying acoustic pressure at $100 \mathrm{~ms}$ PRP. The first column represents Time Domain Signal of the echo received, where the x-axis represents the time y-axis represents the amplitude. The second column represents the corresponding FFT of the time domain signal, where $\mathrm{x}$-axis represents the frequency of the waves emitted from the microbubble and y-axis represents the amplitude of the wave .20 
Figure 3.4: The spectrograms of acoustic behavior of Definity microbubbles at varying acoustic pressure received with $2.25 \mathrm{MHz}$ at $10 \mathrm{~ms}$ PRP of 30 s total treatment times. The $\mathrm{x}$-axis represent frequency and y-axis represent treatment times. Figure 3.4 (A, B, C) show 30s of the time dynamic while Figure 3.4 (D, E, F) magnify the spectrogram to $5 \mathrm{~s}$ in order to clearly observe the signal. (A) and (D) at $\mathrm{PNP}=218 \mathrm{kPa}$;(B) and (E) at $\mathrm{PNP}=335 \mathrm{kPa} ;$ (C) and (F) at

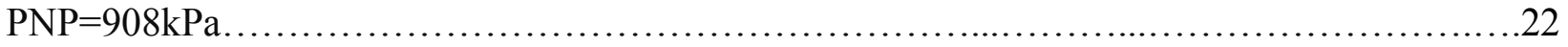

Figure 3.5: The spectrograms of acoustic behavior of Definity microbubbles at varying acoustic pressure received with $2.25 \mathrm{MHz}$ at $100 \mathrm{~ms}$ PRP of $30 \mathrm{~s}$ total treatment times. The $\mathrm{x}$-axis represent frequency and y-axis represent treatment times. Figure 3.5 (A, B, C) show 30s of the time dynamic while Figure 3.5 (D, E, F) magnify the spectrogram to $5 \mathrm{~s}$ in order to clearly observe the signal. (A) and (D) at $\mathrm{PNP}=218 \mathrm{kPa}$;(B) and (E) at $\mathrm{PNP}=335 \mathrm{kPa}$; (C) and (F) at $\mathrm{PNP}=908 \mathrm{kPa}$ 23

Figure 3.6: Integrated cavitation dose of the harmonic and Ultra-harmonic frequency at different acoustic pressures $(218 \mathrm{kPa}, 335 \mathrm{kPa}, 908 \mathrm{kPa})$ received with $2.25 \mathrm{MHz}$ transducer at a PRP of $10 \mathrm{~ms}$ and $100 \mathrm{~ms}$ for $30 \mathrm{~s}$ of treatment time. .25

Figure 3.7: The correlation of MDA-MB-231 breast cancer cells vs. integrated cavitation dose of harmonic (2f, 3f, 4f) and Ultra-harmonic (2.5f, 3.5f, 4.5f) at different pressure $(218 \mathrm{kPa}, 335 \mathrm{kPa}$, $908 \mathrm{kPa}$ ), frequencies received with $2.25 \mathrm{MHz}$ transducer exposed to an 8-cycle pulse sequence with $10 \mathrm{~ms}$ and $100 \mathrm{~ms}$ PRP for a 30 s treatment time. .27 


\section{Chapter 1 Introduction}

\subsection{Background}

Clinically, microbubbles (MB) are utilized in ultrasound (US) imaging as a contrast agent to enhance detection of blood circulation [1]. Experimentally, ultrasound and microbubbles (USMB) have potential applications in enhancing targeted drug delivery of chemotherapeutic drugs and genetic materials [2] and in improving the effectiveness of radiotherapy in cancerous tissues [3,4]. Microbubbles exposed to an ultrasound field can undergo stable linear and nonlinear oscillations, or disruption depending on the ultrasound exposure conditions [3]. The interaction of USMB with cells has been shown to kill cells and enhance plasma membrane permeabilization allowing cell-impermeable molecules to enter a cell, a phenomenon referred to as sonoporation $[5,6]$. The biological mechanism of sonoporation appears to be associated with membrane disruption and enhanced endocytosis mediated by USMB [7]. This dissertation investigates the effect of USMB on cell death and the associated acoustic response under varying ultrasound exposure conditions. 


\subsection{Ultrasound physics}

Ultrasound is a pressure wave with frequencies above $20 \mathrm{kHz}$. An ultrasound wave propagates through tissue through longitudinal oscillation of the particles of the medium about their equilibrium position in the direction of propagation [8]. As a wave propagates through a medium, energy is lost due to absorption and scattering; attenuation is a term used to account for scattering and absorption of a wave. Scattering is a process during which reflected acoustic waves change direction in regards to the incident acoustic wave. The scattering of ultrasound is due to the difference in the acoustic impedance within a medium $[9,10]$. The acoustic impedance of a medium is given by:

$$
Z=\rho c
$$

where $\rho$ is the density of the medium $\left[\mathrm{kg} / \mathrm{m}^{3}\right]$ and $\mathrm{c}$ is the speed of sound $[\mathrm{m} / \mathrm{s}]$.

Absorption is a process during which acoustic wave energy is converted to thermal energy. At high intensity ultrasound conditions, the absorption of ultrasound energy interacts with tissue and cause tissue coagulation; this is referred to as thermal bio-effects [11]. HIFU has been used to treat soft cancer tissue [12]. HIFU causes tissue temperatures to rise in a particular focal area while the surrounding tissue remains unheated. In vivo studies have shown that a $1.7 \mathrm{MHz}$ frequency pulse with an intensity of $1500 \mathrm{~W} / \mathrm{cm}^{2}$ and time exposure of $1-2 \mathrm{sec}$, cause the temperature to exceed $56{ }^{\circ} \mathrm{C}$. This causes coagulative necrosis with a margin of $6-10$ cells between live and dead cells at the focal region $[12,13]$. In addition, ultrasound can cause bioeffects through non-thermal mechanisms such as cavitation, which describes the interaction between acoustic wave and gas bubbles or nucleus $[14,15,16]$. 


\subsection{Ultrasound and Microbubbles}

\subsubsection{Microbubble Agent}

Microbubbles are small in size, less than $10 \mu \mathrm{m}$ in diameter making them smaller than red blood cells. The structure of microbubbles consists of a gas core and an outer shell. The outer shell of micobubbles is composed of a variety of biocompatible materials such as proteins, lipids or polymers, ranging approximating $10-20 \mathrm{~nm}$ in thickness. The core of microbubbles comprise of gas of high molecular weight such as perfluorocarbon, sulphur hexafluoride, and perfluoropentane $[17,18]$.

\subsubsection{Microbubble Physics}

The response of microbubbles in an ultrasound field depend on (1) the MB characteristics (size, gas and shell) and environment, and (2) on the ultrasound exposure parameters such as peak negative pressure (PNP) and pulse center frequency (PCF) [19]. Microbubbles in an ultrasound field can undergo stable oscillation - linear and non-linear - or disruption [20]. The oscillation of MBs is maximized at their resonant frequency [21,22]. The resonant frequency for free gas bubbles, assuming a linear and an undamped system, is:

$$
\mathrm{f}_{\mathrm{r}}=\frac{1}{2 \pi \mathrm{a}} \sqrt{\frac{3 \gamma_{\mathrm{c}}}{\rho} \mathrm{P}_{\mathrm{o}}}
$$

where $a$ is the initial radius of the bubble, $\gamma_{\mathrm{c}}=\gamma_{\mathrm{p}} / \gamma_{\mathrm{v}}$ (where $\gamma_{\mathrm{p}}$ is the heat capacity at constant pressure and $\gamma_{\mathrm{v}}$ at constant volume), $\rho$ is the density of the surrounding medium, and $\mathrm{P}_{\mathrm{o}}$ is the static pressure at the bubble surface [23]. 
As acoustic pressure increases, the microbubbles shift towards nonlinear oscillation [24]. Microbubbles expand during negative pressures and contract during positive pressures. Noninertial cavitation occurs during low pressure and microbubbles oscillate both linearly and nonlinearly about their equilibrium radius [25]. Inertial cavitation occurs at high acoustic pressures where microbubbles undergo large expansion followed by a violent collapse [26]. The microbubbles experiencing inertial cavitation generally reach a radius two times larger than their initial diameter $[17,18]$. The microbubbles undergo inertial cavitation (IC) depending on shell and gas properties. It has been shown that the thick shell has a high threshold compared with thin shell microbubbles. For example, Optison microbubbles are destroyed at $0.13 \mathrm{MPa}$ while Biospheres are destroyed at $0.23 \mathrm{MPa}$ [27]. Furthermore, it has been shown that the threshold increases as center frequency increases in both thin shell microbubbles and thick shell microbubbles. Microbubbles that undergo linear oscillation emit signal frequencies equal to that of driving frequency (fundamental frequency). Bubbles that undergo non linear oscillation emit signal frequencies in the harmonics ( $2 \mathrm{f}, 3 \mathrm{f}, 4 \mathrm{f}$ etc), subharmonic $(\mathrm{f} / 2, \mathrm{f} / 3$ etc) and ultraharmonic $(3 \mathrm{f} / 2,5 \mathrm{f} / 2$, etc) [27]. Figure 1 illustrates the occurrence of harmonic, ultra harmonic and sub harmonic signals after transmitting a pulse at $20 \mathrm{kHz}$ [28]. 


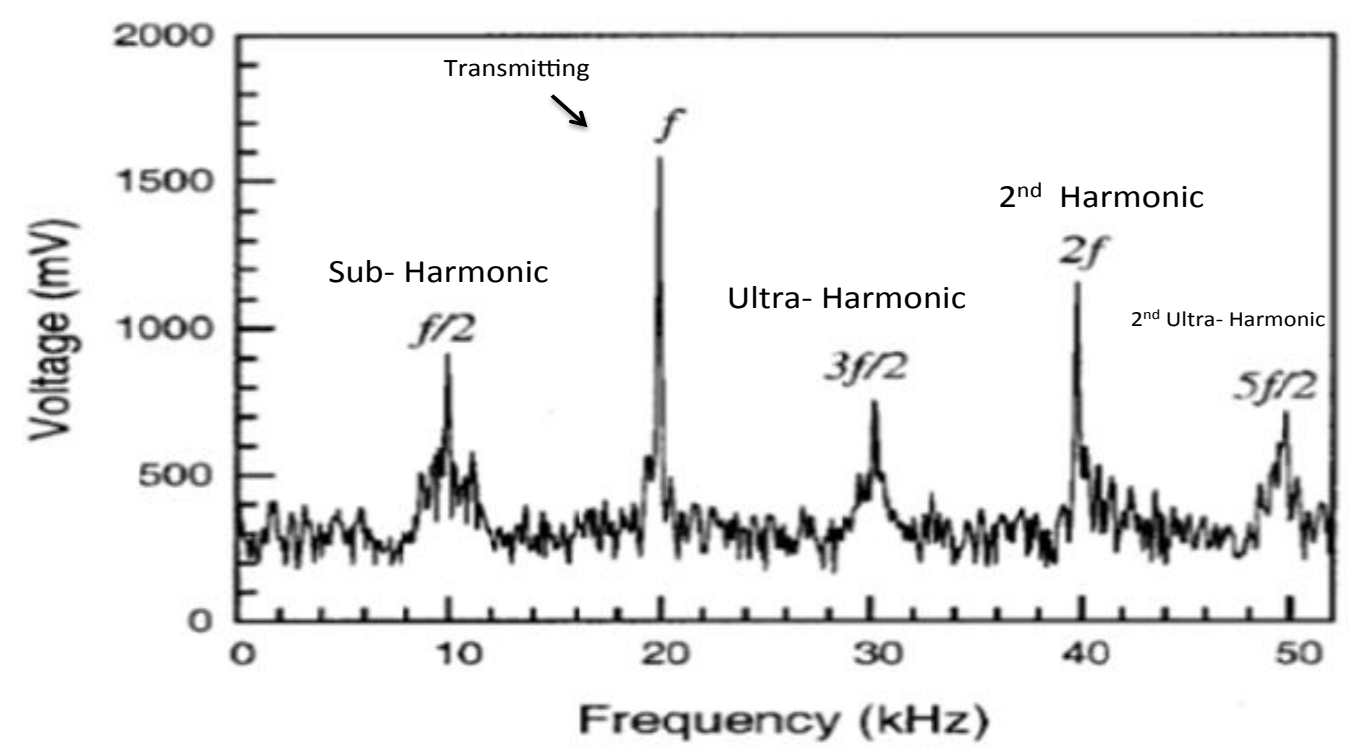

Figure 1.1: The acoustic emission spectrum for bubbles using the passive cavitation detection measurement. The figure includes fundamental signals, 2nd Harmonic, Ultra-Harmonic, 2nd Ultra-Harmonic and Sub-Harmonic [28].

\subsection{Sonoporation}

Sonoporation has recently been studied as new modality for cancer treatment. Ultrasound and microbubbles have the ability to enhance cellular uptake of molecules to target areas. Several investigations have already examined the effect of varying ultrasound parameters on cells and microbubbles, however there is limited understanding on the correlation between microbubbule acoustic response during sonoporation and cell viability. Studies have reported on the impact of different ultrasound parameters on cell membranes [29,30,31]. Sonoporation has been used for cancer treatment but it remains a challenge to achieve consistent and controlled delivery of drugs to the interior of tumor. Furthermore, the acoustic dynamic of sonoporation remains unclear. Sonoporation is dependent on ultrasound exposure parameters including: frequency, acoustic pressure, pulse repetition period and pulse duration. One investigation has indicated that an optimal ultrasound condition for sonoporating endothelial cells is $30 \mathrm{~s}$ [32]. Generally, as 
acoustic pressure and pulse repetition frequency increase, both cell permeability and cell death increases [33].

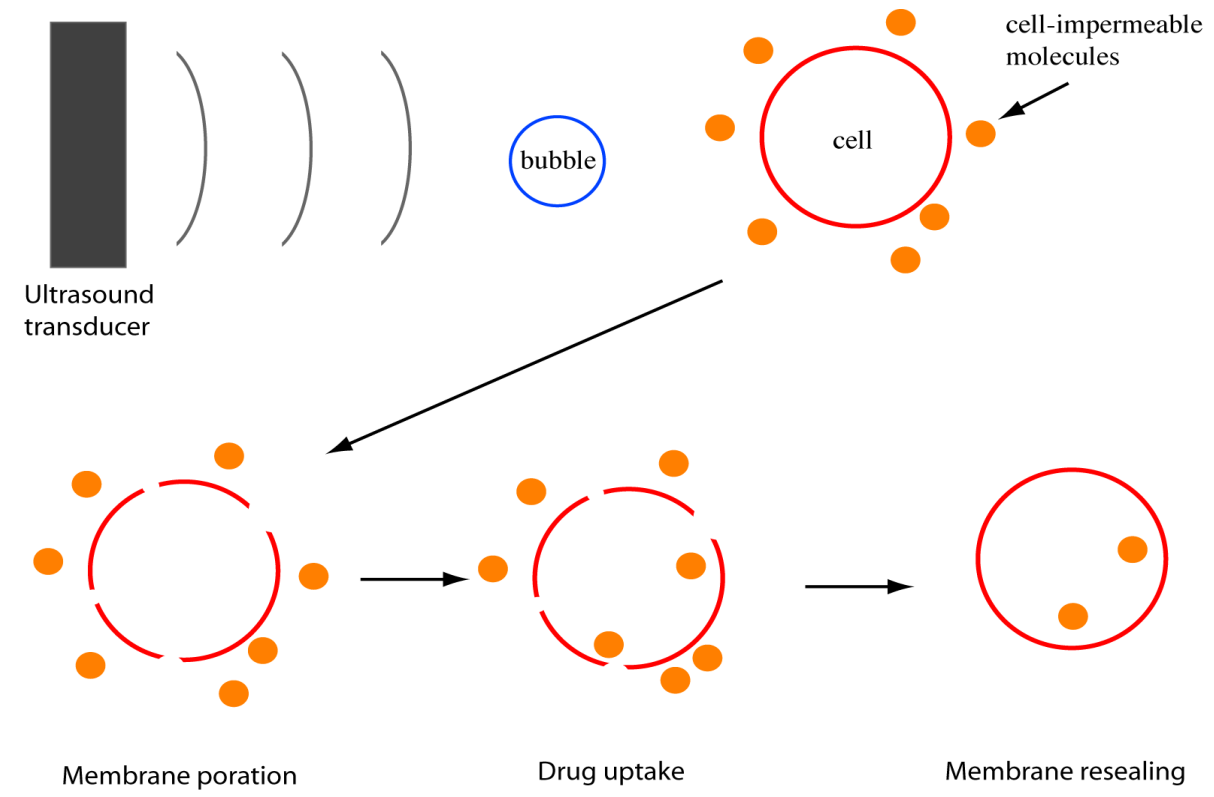

Figure 1.2: Schematic diagram of ultrasound and microbubble induced sonoporation by cell membrane disruption [33]. 


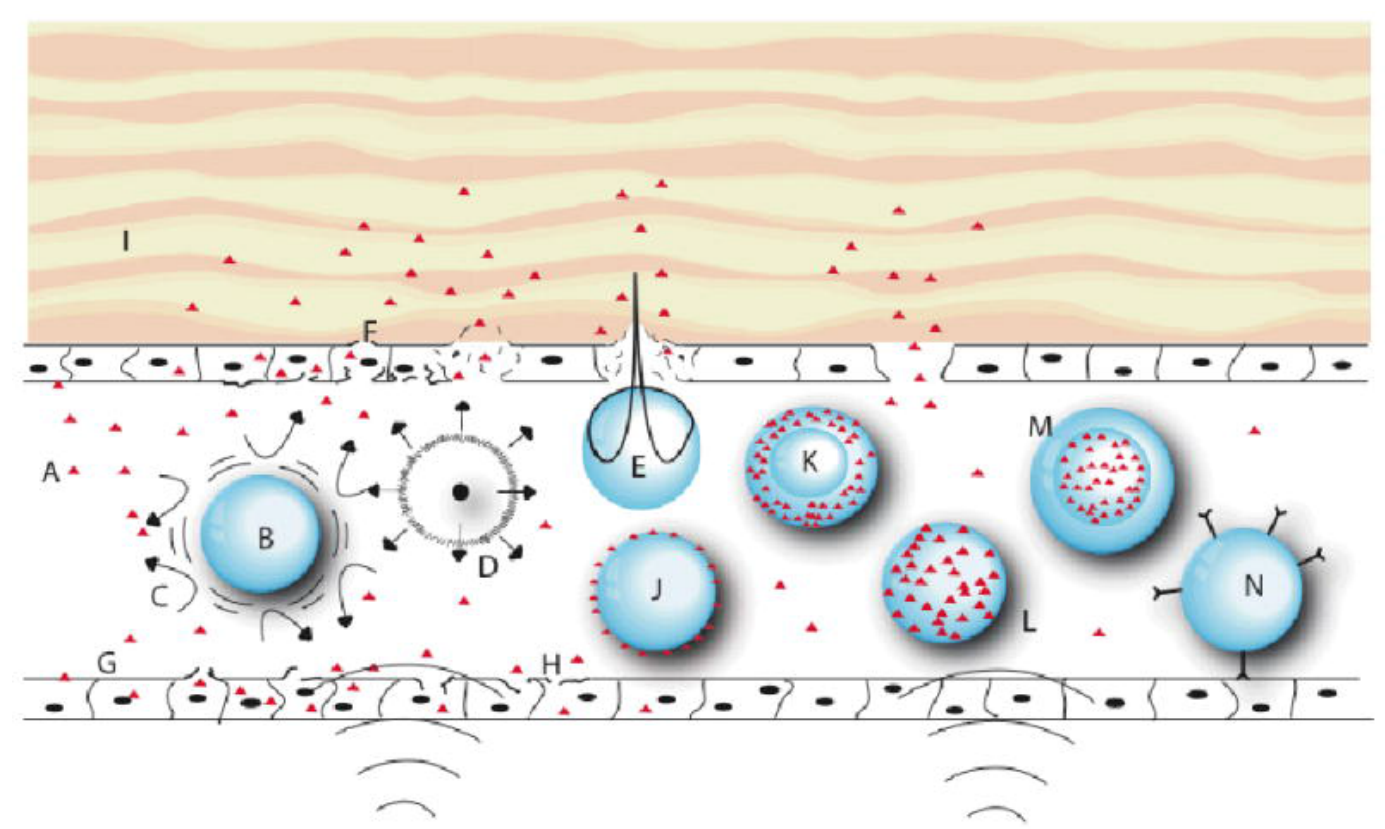

Figure 1.3: The acoustical mechanisms associated with ultrasonically-stimulated microbubbles include microstreaming induced by oscillating microbubbles, shock waves due to non-stable cavitation, and micro-jet formation due to collapsing microbubbles. A: drug molecules; B: stable cavitation of microbubbles; C: Microstreaming; D: non stable cavitation of microbubbles produce shock waves; E: fluid microjet; F: cell membrane disruption; G: non ruptured cell membrane with increase permeability due to USMB; H: cell membrane damage due to shock waves and microstreaming; I: extravascular tissue; J: microbubble loaded with drug molecules on its surface; K: microbubbles loaded with drug molecules in lipophilic phase; L: micelle loaded with drug molecule lipophilic phase; M: liposome with aqueous interior; $\mathrm{N}$ : targeted microbubble [20].

Cavitation has been associated with sonoporation-mediated delivery of drugs and genetic materials [18]. Stable oscillation of microbubbles has been shown to generate microstreaming within the surrounding fluid and induce shear stress on nearby biological membranes [47]. In addition, microbubbles undergoing inertial cavitation can generate shockwaves and fluid microjets and induce bioeffects on biological cells [7]. Furthermore, microbubbles can be loaded with molecules and targeted to a diseased site, and subsequently releasing the drug molecules by disrupting the microbubbles acoustically (Figure 1.3). 


\subsection{Microbubble Acoustic Response}

\subsubsection{Microbubble Characterization}

Microbubbles can be characterized acoustically using active and passive detection methods. Active cavitation detector uses a pulse-echo technique, which transmits and receives ultrasound pulses using the same transducer [34]. In passive cavitation detection (PCD), one transducer emits the pressure wave while the other transducer is set only in receive mode, which listens to the signal emissions from microbubble passively [35].

\subsubsection{Cavitation Dose}

Cavitation in this study refers to the acoustic activity of micorbubbles exposed to an ultrasound field. Cavitation dose is important in characterizing microbubble acoustic response; it quantifies the amount of energy emitted by USMB [36]. Integrated cavitation dose quantifies the cavitation activity of microbubbles when exposed to ultrasound pulses. The integrated cavitation dose has been related to various bio-effects induced by ultrasound and microbubbles [37]. One of the specific aims in this project is to measure the integrated cavitation doses. The frequency spectrum of the signal emitted by microbubbles is calculated using:

$$
S(f, \tau)=\int_{-\infty}^{+\infty} s(t, \tau) e^{-j 2 \pi f t} \cdot d t
$$

where $s(t, \tau)$ is the time domain signal and $S(f, \tau)$ is the frequency spectrum of $s(t)$; $t$ refers to the fast time and $\tau$ refers to the slow time of the ultrasound exposure pulse sequence.

The cavitation dose (CD) was calculated using Equation 1.4 over a certain frequency range at each of the slow time-points $\tau$ : 


$$
C D(\tau)=\int_{f n+\Delta}^{f n-\Delta f}\left|\mathrm{~S}_{\mathrm{i}}(f, \tau)\right| d f
$$

The integrated cavitation dose over the exposure duration was calculated using;

$$
I C D=\int_{0}^{I T} C D(\tau) d \tau
$$

Where IT is the insonation time of the ultrasound pulse sequence.

\subsection{Thesis Outline}

This project aims to study USMB-induced cell death through the characterization of microbubble acoustic response. In this study, the effect on cell viability was associated to harmonic cavitation doses in an effort to better understand USMB induced cell death. This study is guided by the hypothesis that the integrated cavitation dose is correlated with cell death when treated with USMB. The specific objectives of this investigation are:

1- To determine the effects of peak negative pressure and pulse repetition period on cell viability and acoustic response of microbubbles measured by PCD (Passive Cavitation Detection)

2- To investigate the relationship between cell viability and inertial cavitation dose. 


\section{Chapter 2 Materials and Methods}

\subsection{Introduction}

Cells and microbubbles in suspension were exposed to varying ultrasound exposure conditions. During ultrasound exposure, the microbubble acoustic response was measured using a passive cavitation detector (PCD). Following ultrasound exposure cell viability was measured using flow cytometry.

\subsection{Cell model}

A human adenocarcinoma breast cell line (MDA-MB-231- ATCC, MD, USA) was used in this project. MDA cells were grown in RPMI media with 5\% streptomycin antibiotic and $10 \%$ fetal bovine serum. The cells were grown as a monolayer in $175 \mathrm{~cm}$ tissue culture flasks with humidity of $5 \% \mathrm{CO}_{2}$ at $37{ }^{\circ} \mathrm{C}$. The cells were exposed to USMB (ultrasound and mirobubbles) at a concentration of $1 \times 10^{6}$ cells $/ \mathrm{mL}$ at a volume of $1 \mathrm{~mL}$. Cells were suspended in RPMI cell culturing media at room temperature during the experiment. 


\subsection{Ultrasound exposure system}

The system consists of an arbitrary wave generator (AWG610, Tektronix, USA), power amplifier (AG series Amplifier, T\&C power conversion, Inc., USA) and AlazarDso acquisition system. Two transducers attached to micropositioning systems were used in this project: Transmitting transducer was $0.5 \mathrm{MHz}$ (IL0508HP, Valpey Fisher Inc, Hopkinton,MA) with a diameter of 25.4 $\mathrm{mm}$, a focal length of $4.3 \mathrm{~cm}$ and a beamwidth of $9.2 \mathrm{~mm}$; receiving transducer was $2.25 \mathrm{MHz}$ (IL0208HP, Valpey Fisher Inc, Hopkinton, MA) with a diameter of $25.4 \mathrm{~mm}$ and bandwidth of $30 \%$ at $-6 \mathrm{~dB}$. The passive cavitation detection transducer was positioned at $90^{\circ}$ in relation to the transmitting transducer. The ultrasound apparatus held the sample submerged in degassed water inside a tank with dimensions $50 \times 30 \times 20 \mathrm{~cm}^{3}$. A schematic diagram of the ultrasound apparatus is shown in Figure 2.1. The sample was placed at the intersection of the focal zones of the transducers in a cylindrical treatment chamber with $8 \mathrm{~mm}$ internal diameter. Focusing was done using a micropositioning system with a needle to receive the pulse-echo from the needle.

The effect of the following ultrasound exposure parameters on viability and microbubble response was investigated at varied pulse repetition periods (PRP) of 10ms and 100ms over different pressures $(218,335$ and $908 \mathrm{kPa})$ The acoustic pressure was measured in the absence of cell exposure chamber using a needle hydrophone (Precision Acoustics Ltd, Dorchester, and Dorset, UK), 8 cycles tone-burst pulse (pulse duration of $16 \mu \mathrm{s}$ ) and a total insonation time of 30s. The trigger delay in the acquisition system was set to $70 \mu \mathrm{s}$. The acoustic emission from MBs acquired using the passive cavitation detection was analyzed using FFT spectral analysis (MATLAB) and cavitation dose calculations [38]. 


\subsection{Cavitation dose}

The Power Spectral Density (PSD) was calculated in MATLAB to understand the strength of a signal distribution in the frequency domain (FFT). This was implemented in MATLAB using equation 2.1. Spectrograms were used to analyze the microbubbles' response throughout the entire treatment time.

$$
S(f, \tau)=20 \log \operatorname{FFT}[(s(t, \tau)]
$$

Where $t$ is the fast time and $\tau$ is the slow time.

The cavitation dose was calculated using Equation 2:

$$
\mathrm{CD}(\tau)=\sum_{f_{n}-\Delta f^{\prime}}^{f_{n}+\Delta f^{\prime}}|\mathrm{S}(\mathrm{f}, \tau)| \Delta f
$$

Where $\mathrm{S}(\mathrm{f}, \tau)$ is the FFT of $\mathrm{s}(\mathrm{t}), \Delta \mathrm{f}=0.012 \mathrm{MHz}$ is the step size in frequency domain, $\Delta \mathrm{f}^{\prime}=$ $0.1 \mathrm{MHz}$, and $\mathrm{f}_{\mathrm{n}}=1.00,1.50$ and $2.00 \mathrm{MHz}$ at the harmonic frequencies and 1.25, 1.75 and 2.25 $\mathrm{MHz}$ at the ultra-harmonic frequencies. The $\mathrm{CD}$ as a function of $\tau$ represents the $\mathrm{MB}$ signal at each ultrasound pulse within the sequence.

The calculation of integrated cavitation dose was performed using the ICD equation below:

$$
I C D=\sum_{1}^{\tau_{i}} C D . \Delta \tau
$$

Where $\tau_{i}$ is 3000 and $\Delta \tau=10 \mathrm{~ms}$ for the $10 \mathrm{~ms}$ PRP, and $\tau_{i}$ is 300 and $\Delta \tau=100 \mathrm{~ms}$ for the 100ms PRP [45]. 


\subsection{Microbubble contrast agent}

Definity Microbubbles (Lantheus Medical Imaging, North Billerica, MA0186, USA), a clinically approved ultrasound contrast agent, were used. The microbubbles comprised of a phospholipid shell encapsulating gaseous octafluoropropane $\left(\mathrm{C}_{3} \mathrm{~F}_{8}\right)$ with a mean diameter of 1.1-3.3 $\mu \mathrm{m}$ to a maximum diameter of $20 \mu \mathrm{m}$ according to the manufacturer. Definity microbubbles had an initial

concentration of $1.2 \times 10^{10}$ bubbles/mL, which was activated by shaking with Vial-Mix (Lantheus Medical Imaging Inc) for 45 seconds. The microbbbules concentration used in this experiment per sample was $33 \mu \mathrm{L} / \mathrm{mL}$. Microbubbles were diluted with PBS at 1:1 and 1:4 ratios, respectively.

\subsection{Treatment procedure}

Cell samples were placed in the chamber and treated with ultrasound pulses in the presence of microbubbles. Microbubbles were added just prior to ultrasound exposure. During ultrasound treatment, the cells were uniformly distributed using gentle magnetic stirring. The chamber was washed between exposures using PBS.

\subsection{Cell viability}

Following treatment with ultrasound and microbubbles, cell viability was measured using flow cytometry (BD FACSCalibur; San Jose, CA, USA) and propiduim iodide (PI) staining. Cells unstained with PI were referred to as viable. Cells stained with PI were considered non-viable; PI stain cells whose membrane integrity has been comprised. Dilute Propidium Iodide (PI), 5 $\mu \mathrm{L} /$ samples, was made by 1:4 dilutions with PBS. 


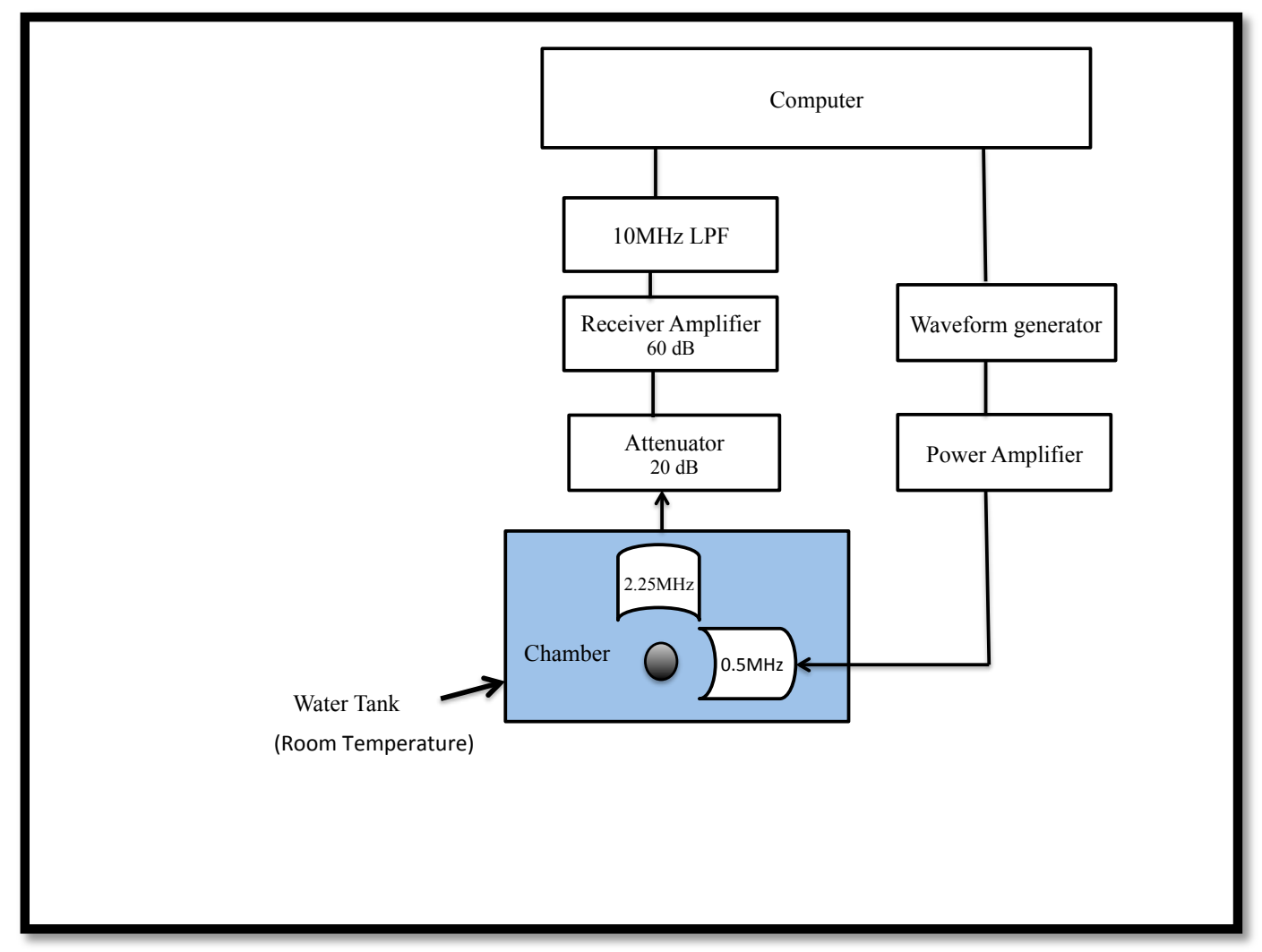

Figure 2.1: The schematic diagram of the ultrasound exposure system. Microbubbles and cells were exposed in a cylindrical chamber with an internal diameter of $8 \mathrm{~mm}$ and a height of _90 $\mathrm{mm}$; _30_mm was covered with acoustic membrane. Microbubbles were exposed to an ultrasound field using a $0.5 \mathrm{MHz}$ transducer and emissions were received using $2.25 \mathrm{MHz}$ transducer. Harmonic and ultra-harmonic frequencies were detected. 


\section{Chapter 3 Results}

\subsection{Introduction}

This project investigated the response of both MDA-Mb-231 cells and Definity microbubbles when exposed to varying acoustic pressures $(0-908 \mathrm{kPa})$ and pulse repetition period (10ms and $100 \mathrm{~ms})$, with $16 \mu \mathrm{s}$ pulse duration and a microbubbble concentration of $(3.3 \% \mathrm{v} / \mathrm{v})$ for an insonation time of 30s. Cell viability results are shown in section 3.1, the acoustic characteristics of microbubbles in section 3.2 and the relationship between cell viability and integrated cavitation dose in section 3.3 .

\subsection{Non-viable cells}

Figure 3.1 illustrates the effects of acoustic pressure and pulse repetition period on cell viability. The viability of a cell when exposed to ultrasound and microbubbles is influenced by both peak negative pressure and pulse repetition period. The percentage of non-viable cells significantly increases with peak negative pressure and only slightly decreases with pulse repetition period. In terms of peak negative pressure, there were statistical differences between the control $(0 \mathrm{kPa}$, $2.3 \%$ cell death), and all the pressures $(218 \mathrm{kPa}, 335 \mathrm{kPa}, 908 \mathrm{kPa})$ for both $10 \mathrm{~ms}$ and $100 \mathrm{~ms}$. Cell death at $218 \mathrm{kPa}(14 \%$ at $10 \mathrm{~ms} \mathrm{PRP}$, and $11 \%$ at $100 \mathrm{~ms} \mathrm{PRP})$ and $335 \mathrm{kPa}(10 \mathrm{~ms}=16 \%$, $100 \mathrm{~ms}=14 \%$ ) are similar with no significant differences found between these pressures. The 
maximum amount of cell death observed for pulse repetition periods of both $10 \mathrm{~ms}$ and $100 \mathrm{~ms}$ was found at $908 \mathrm{kPa}$ with $26 \%$ and $22 \%$, which was also statistically higher than both $218 \mathrm{kPa}$ and $355 \mathrm{kPa}$ treatments. In addition, the cell viability was not statistically different between the pulse repetition periods of $10 \mathrm{~ms}$ and $100 \mathrm{~ms}$.

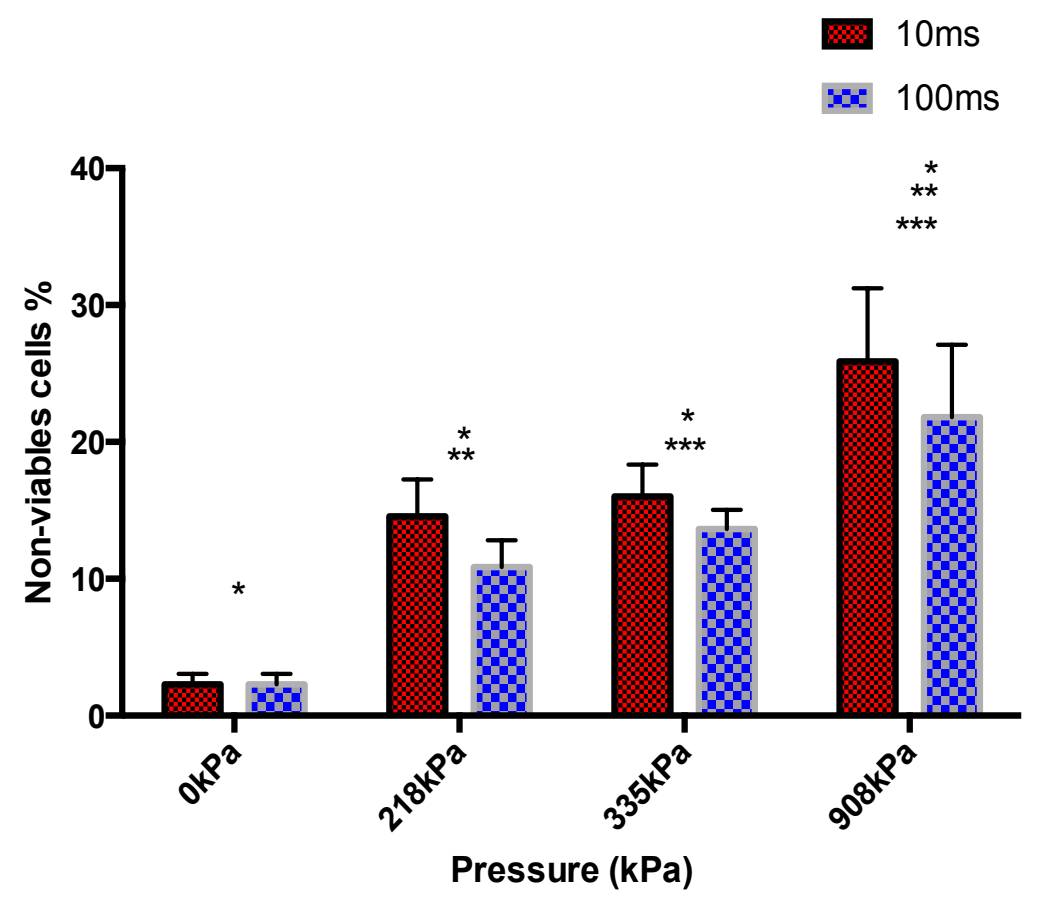

Figure 3.1: Non-viable cell of MDA-MB-231 cells exposed to a $0.5 \mathrm{MHz}$ center frequency at varying acoustic pressures $(218 \mathrm{kPa}, 335 \mathrm{kPa}, 908 \mathrm{kPa})$ with a $10 \mathrm{~ms}$ (black bars) and 100ms (grey bars) PRP and total treatment time of 30s in 3.3\% Definity volume concentration. The percentages of Non-viable cells are shown with respect to different pressures at center frequencies of $0.5 \mathrm{MHz}$.

\subsection{Acoustic characteristics of Microbubbles}

The effect of acoustic pressure (PNP) on acoustic MB behaviors is shown in Figures 3.2-to-3.5. The acoustic behavior of a $\mathrm{MB}$ exposed to 8 cycles at $0.5 \mathrm{MHz}$ frequency pulse at acoustic pressures $(218 \mathrm{kPa}, 335 \mathrm{kPa}, 908 \mathrm{kPa})$ for $30 \mathrm{~s}$ depends on the applied PNP. Spectrograms associated with the acoustic response time domain signal and the FFT of the MB at 10ms and 
100ms pulse repetition period are presented in Figure 3.2 and 3.3 respectively, and the FFT spectrograms are shown in Figure 3.4 and 3.5, respectively.

\subsubsection{Effect of peak negative pressure}

The time domain signals received with the $2.25 \mathrm{MHz}$ transducer and their corresponding frequency spectra are shown in Figure 3.2 at 10ms pulse repetition period. As the pressure of the transmitted wave was increased, the signal from the MBs increased as shown in Figure 3.2 (A, B and C). As seen in Figure 3.2 (A), the amplitude of the time domain signal is 1, and as pressure increases up to $908 \mathrm{kPa}$ the amplitude increases to 1.5 . At $218 \mathrm{kPa}$, the MBs experience weak nonlinear oscillation emitting the fundamental (f), harmonic signals (2f, $3 \mathrm{f}, 4 \mathrm{f}$ and $5 \mathrm{f}$ ) and ultraharmonic signals (1.5f, 2.5f, 3.5f and 4.5f) as shown in Figure 3.2(D). At 335kPa, the MBs strong non-linear oscillation with an emission of the fundamental (f), harmonic signals ( $2 \mathrm{f}, 3 \mathrm{f}, 4 \mathrm{f}, 5 \mathrm{f}$ and $6 \mathrm{f}$ ) and ultra-harmonic signals (1.5f, 2.5f, $3.5 \mathrm{f}$ and $4.5 \mathrm{f}$ ) shown in Figure 3.2(E). At 908kPa, the MBs undergo irregular compression emitting the fundamental (f), harmonic signals (2f, $3 \mathrm{f}, 4 \mathrm{f}, 5 \mathrm{f}$ and 6f) and ultra-harmonic signals (1.5f, 2.5f, 3.5f, 4.5f) shown in Figure 3.2(F). Also, in Figure 3.2 (C) the emission spectrum from the MB appears to broaden at higher pressures indicating increased frequency emissions associated with non-linearity. In comparing the FFT graphs of the different pressures it is also notable that the amplitude of the harmonic signals increases on average, as the pressure increases (Figure 3.2 D, E, F). For example, the amplitude of the second harmonics (2f) increases from $\sim 350$ at $218 \mathrm{kPa}$ to $\sim 620$ at $335 \mathrm{kPa}$. The second harmonics increases further when the pressure goes up to $908 \mathrm{kPa}(\sim 700)$. At the higher pressures a higher amplitude harmonic and sub-harmonic signals are generated. 

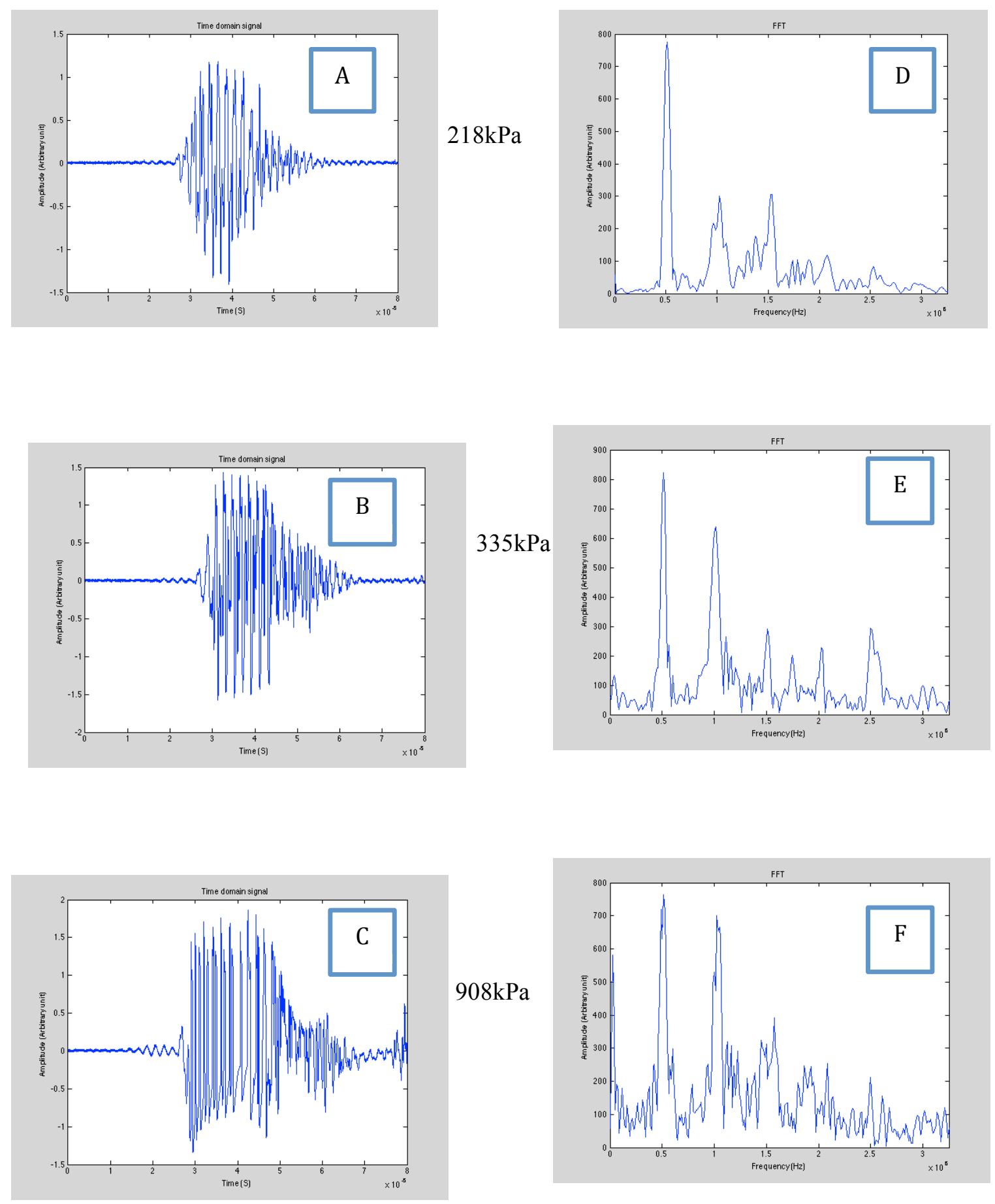

Figure 3.2: The acoustic behavior of Definity microbubbles at varying acoustic pressure at $10 \mathrm{~ms}$ PRP. The first column represents Time Domain Signal of the echo received, where the x-axis represents the time $y$-axis represents the amplitude. The second column represents the corresponding FFT of the time domain signal, where x-axis represents the frequency of the waves emitted from the microbubble and the y-axis represents the amplitude of the wave. 
The time domain signals received with the $2.25 \mathrm{MHz}$ transducer and their corresponding FFT spectra are shown in Figure 3.3 at $100 \mathrm{~ms}$ pulse repetition period. As the pressure of the transmitted wave was increased, the echo signal was also increased as shown in Figure 3.3(A, B, C). At $218 \mathrm{kPa}$, the MBs also experience weak non-linear oscillation emitting the fundamental (f), harmonic signals (2f, $3 f$, $4 f$ and $5 f$ ) and ultra-harmonic signals (1.5f, 2.5f, $3.5 f$ and $4.5 f)$ seen in Figure 3.3(D). At 335kPa, the MBs strong non-linear oscillation with an emission of the fundamental (f), harmonic signals (2f, $3 f$, $4 f, 5 f$ and $6 f)$ and ultra-harmonic signals (1.5f, $2.5 f$, $3.5 \mathrm{f}$ and $4.5 \mathrm{f}$ ) shown in Figure 3.3(E). Similar to $335 \mathrm{kPa}$, MBs exposed to $908 \mathrm{kPa}$ also exhibit strong non-linear oscillation emitting the fundamental (f), harmonic signals ( $2 \mathrm{f}, 3 \mathrm{f}, 4 \mathrm{f}, 5 \mathrm{f}$ and $6 \mathrm{f}$ ) and ultra-harmonic signals (1.5f, 2.5f, 3.5f and 4.5f) shown in Figure 3.3(F). It is important to note that though the harmonic signals remain the same as those received from the tests with a 10ms PRP, the amplitudes of the signals not including the fundamental signal are lower with 100ms. In comparing the FFT graphs of the different pressures the amplitude of the harmonic signals seem to decreases as the pressure increases (Figure 3.3 D, E, F). When looked at the first harmonic (2f) at $218 \mathrm{kPa}$ the amplitude appears to be at $\sim 380$, drops slightly at $335 \mathrm{kPa}$ and then down to $\sim 310$ at $908 \mathrm{kPa}$. It is evident that higher pressures produce more harmonic and subharmonic signals. 

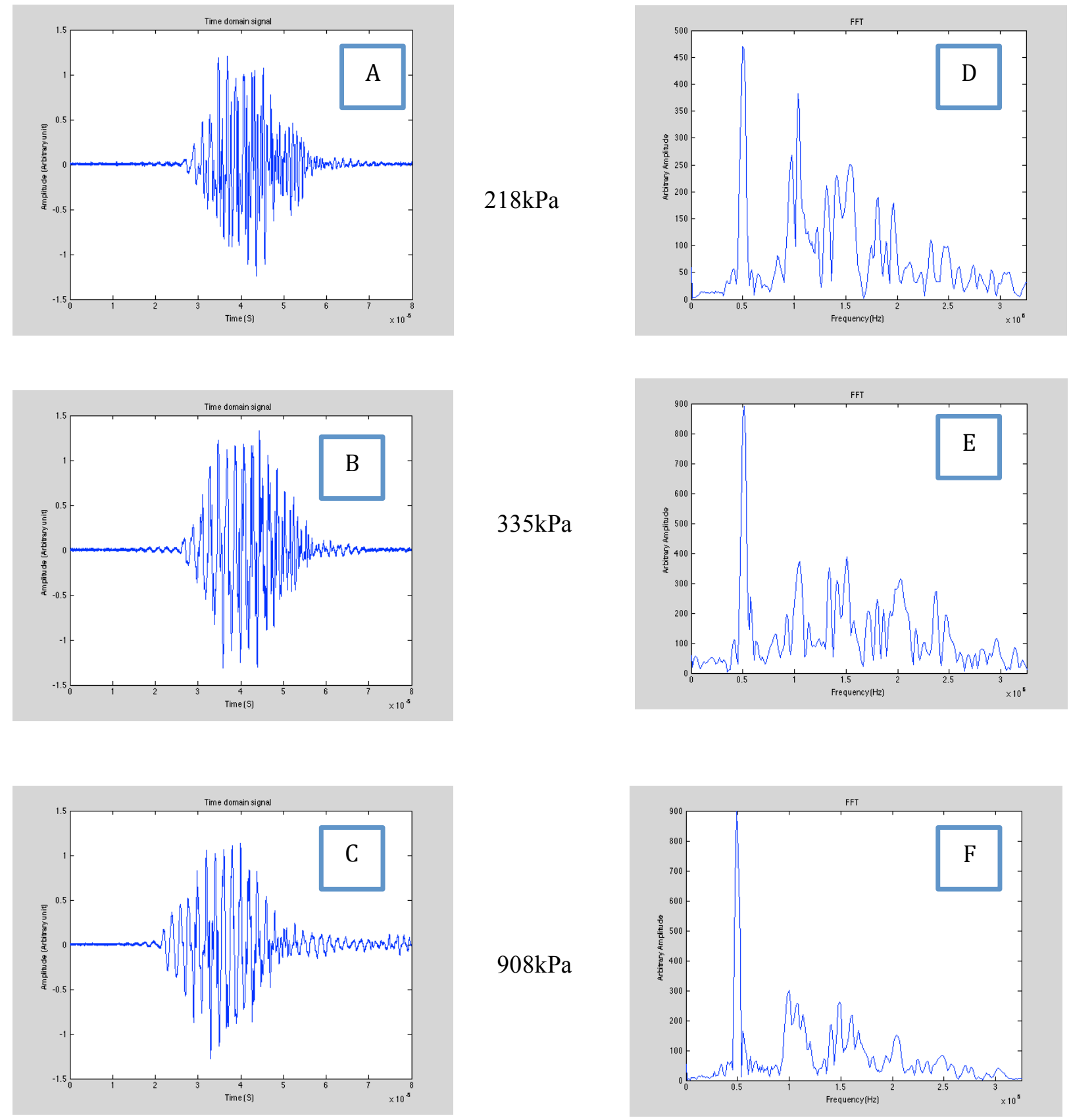

Figure 3.3: The acoustic behavior of Definity microbubbles at varying acoustic pressure at 100ms PRP. The first column represents Time Domain Signal of the echo received, where the x-axis represents the time $y$-axis represents the amplitude. The second column represents the corresponding FFT of the time domain signal, where $\mathrm{x}$-axis represents the frequency of the waves emitted from the microbubble and $y$-axis represents the amplitude of the wave. 
The spectrogram in Figure 3.4 present the time dynamics of the MBs acoustic behavior exposed to a pulse with a pulse repetition period of $10 \mathrm{~ms}$. Figure 3.4 (A, B, C) show $30 \mathrm{~s}$ of the time dynamic while Figure 3.4 (D, E, F) magnify the spectrogram to $5 \mathrm{~s}$ in order to clearly observe the signal. The non-linear oscillation of microbubbles appears to increase as the pressure of the pulse increased from $218 \mathrm{kPa}$ to $908 \mathrm{kPa}$. The time dynamic results suggest that MBs experience strong non-linear oscillation within the first few seconds at each pressure and then undergo a loss of signal (Fig. 3.4). At 218kPa, the loss of signal from the MB appears to occur approximately between $4-5$ s with clear harmonic signals suggesting weakly oscillating microbubbles (Fig. 3.4 A, D). At $335 \mathrm{kPa}$, the spectrograms show additional harmonics as well as a clean MB signal up to $1 \mathrm{~s}$ after the start of the signal (Fig. 3.4 B, E). The shorter oscillation signal compared to 218 $\mathrm{kPa}$ implies that the MB experienced stronger non-linear oscillation. At $908 \mathrm{kPa}$, the $\mathrm{MB}$ signal was lost within milliseconds during exposure (Fig. $3.4 \mathrm{C}$, D). The signal recorded on the spectrograms from Fig. $3.4 \mathrm{~F}$ was slightly cluttered in comparison to the other pressure spectrograms. The results show that as the pressure of the pulse increases, the amount of time for the loss of MB signal decreases. 

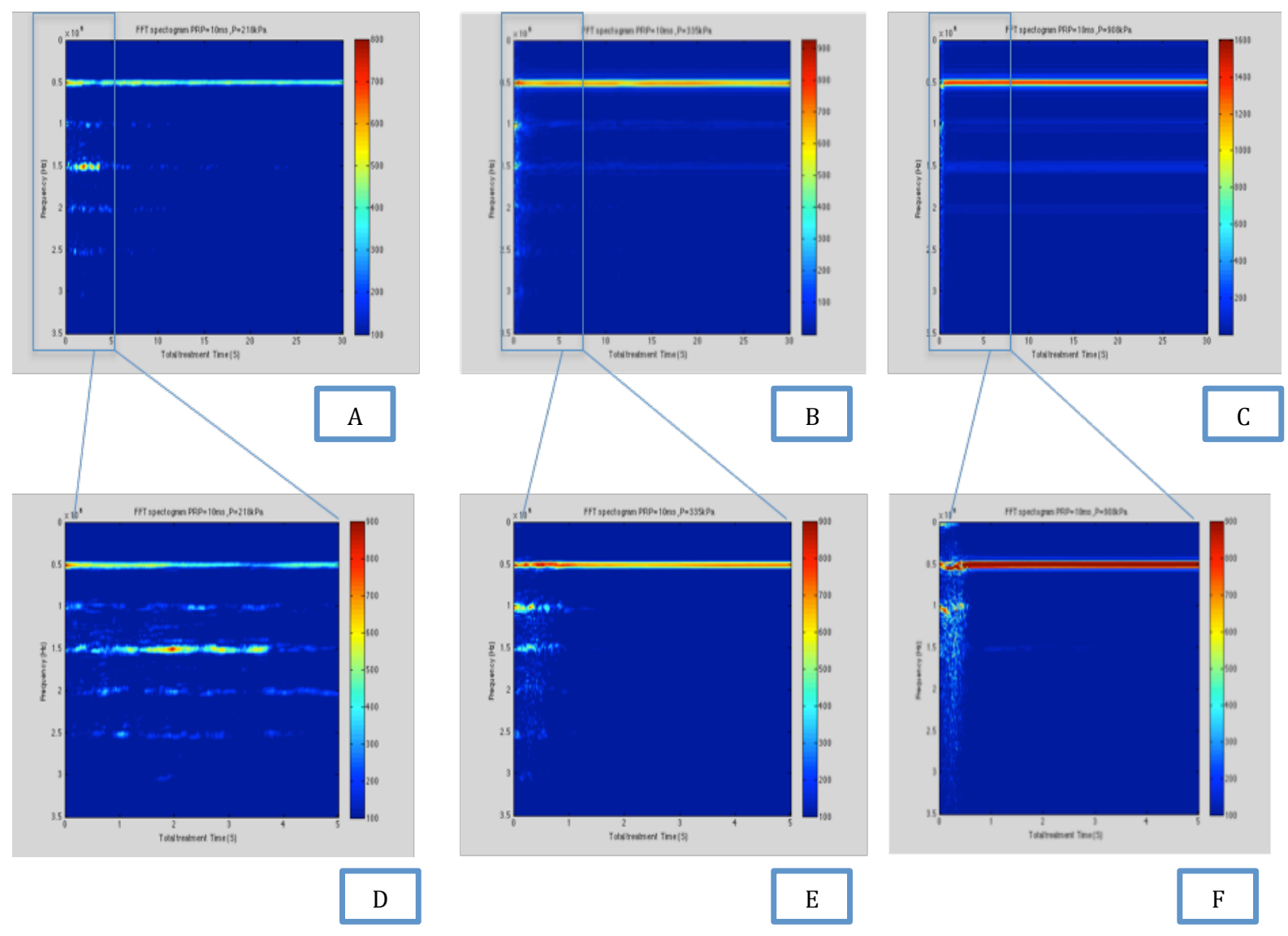

Figure 3.4: The spectrograms of acoustic behavior of Definity microbubbles at varying acoustic pressure received with $2.25 \mathrm{MHz}$ at $10 \mathrm{~ms} \mathrm{PRP}$ of $30 \mathrm{~s}$ total treatment times. The $\mathrm{x}$-axis represent frequency and $\mathrm{y}$-axis represent treatment times. Figure 3.4 (A, B, C) show 30s of the time dynamic while Figure 3.4 (D, E, F) magnify the spectrogram to $5 \mathrm{~s}$ in order to clearly observe the signal. $(\mathrm{A})$ and $(\mathrm{D})$ at $\mathrm{PNP}=218 \mathrm{kPa} ;(\mathrm{B})$ and $(\mathrm{E})$ at $\mathrm{PNP}=335 \mathrm{kPa} ;(\mathrm{C})$ and $(\mathrm{F})$ at $\mathrm{PNP}=908 \mathrm{kPa}$.

The spectrogram in Figure 3.5 present the time dynamics of the MBs acoustic behavior exposed to a pulse with a pulse repetition period of $100 \mathrm{~ms}$, received with a $2.25 \mathrm{MHz}$ transducer. Figure 3.5 (A, B, C) show 30s of the time dynamic while Figure 3.5 (D, E, F) magnify the spectrogram to $5 \mathrm{~s}$ in order to clearly observe the signal. The non-linear behavior of microbubbles appears to increase as the pressure of the pulse in increased from $218 \mathrm{kPa}$ to $908 \mathrm{kPa}$. The time dynamic 
results suggest that the MBs experience strong non-linear oscillation within the first few seconds at each pressure (Fig. 3.5). At $218 \mathrm{kPa}$, the intensity of the signal is low and the signal appears to dissipate at $\sim 10$ s during the exposure. The harmonics from the microbubble suggest that undergo weak non-linear oscillation (Fig. $3.5 \mathrm{~A}, \mathrm{D}$ ). At $335 \mathrm{kPa}$, the spectrograms show additional harmonics as well as a signal up to $25 \mathrm{~s}$ into the exposure and lost thereafter (Fig. $3.5 \mathrm{~B}$, E). Based on the harmonics and the increased cluttering of the signal the MB seems to undergo strong nonlinear oscillation. At $908 \mathrm{kPa}$, the signal from the $\mathrm{MB}$ did not appear to be lost during the entire 30s time frame indicating continued oscillation from the MB (Fig. 3.5 C, F).
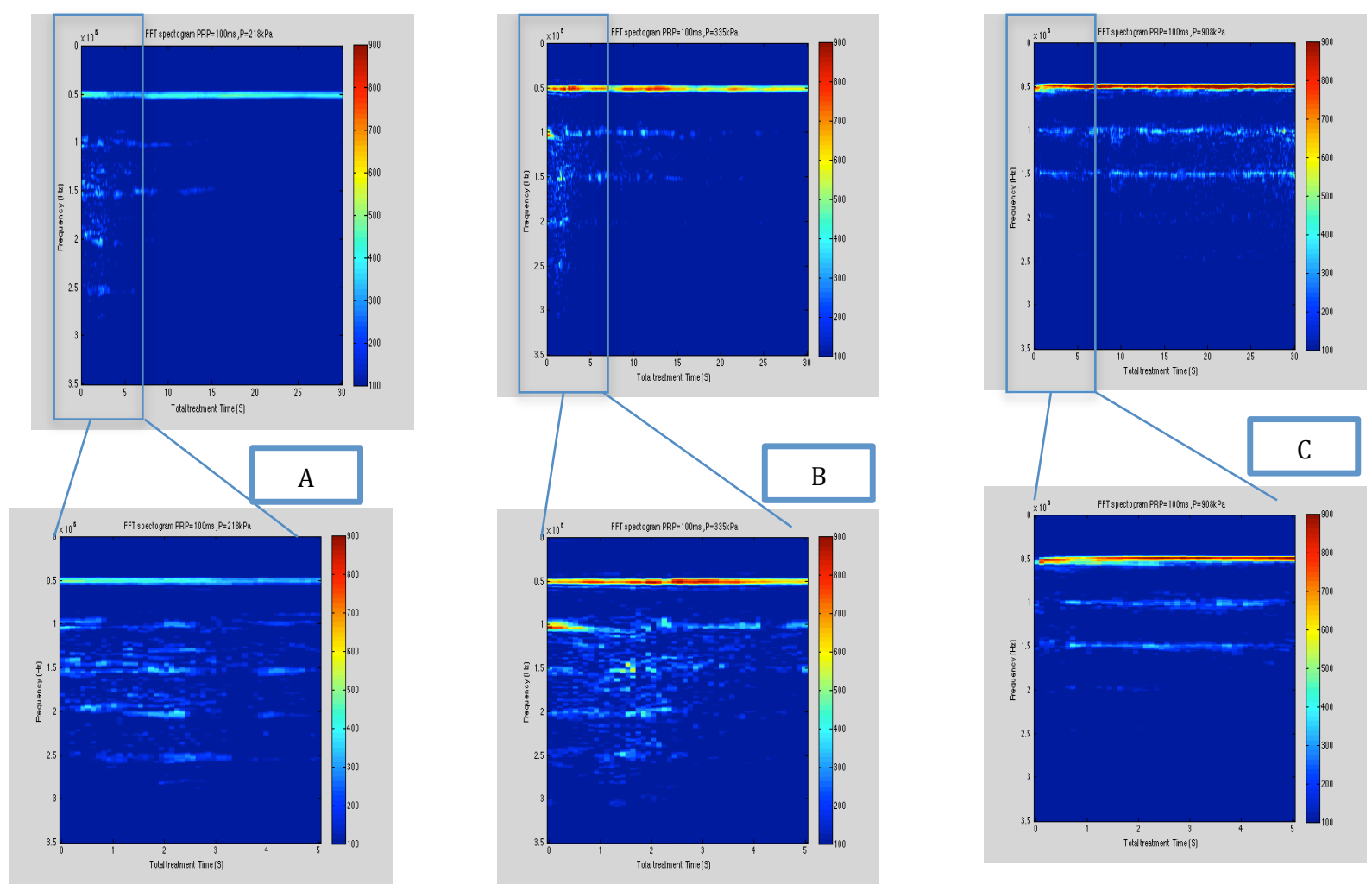

Figure 3.5: The spectrograms of acoustic

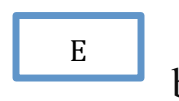
behavior of Definity microbubbles at varying acoustic pressure received with $2.25 \mathrm{MHz}$ at $100 \mathrm{~ms}$ PRP of $30 \mathrm{~s}$ total treatment times. The $\mathrm{x}$-axis represent frequency and y-axis represent treatment times. Figure 3.5 
(A, B, C) show 30s of the time dynamic while Figure 3.5 (D, E, F) magnify the spectrogram to $5 \mathrm{~s}$ in order to clearly observe the signal. (A) and (D) at $\mathrm{PNP}=218 \mathrm{kPa}$;(B) and (E) at $\mathrm{PNP}=335 \mathrm{kPa}$; (C) and (F) at PNP=908kPa.

\subsubsection{Integrated cavitation doses}

Integrated cavitation doses of the received $\mathrm{MB}$ acoustic signal after exposed to 8 cycles, $0.5 \mathrm{MHz}$ center frequency pulsed wave at 10ms and 100ms PRPs are shown in Figure 3.6. The integrated cavitation dose (ICD) increases with acoustic pressure for all harmonic and ultra-harmonic frequency ranges. The ICD values at $10 \mathrm{~ms}$ and $100 \mathrm{~ms}$ PRP increased significantly among both harmonic $(1 \mathrm{MHz}, \quad 1.5 \mathrm{MHz}, \quad 2 \mathrm{MHz})$ and ultra-harmonic $(1.25 \mathrm{MHz}, \quad 1.75 \mathrm{MHz}$ and $2.25 \mathrm{MHz}$ ) frequencies between $218 \mathrm{kPa}$ and $908 \mathrm{kPa}$; the maximum ICD was observed at $908 \mathrm{kPa}$. At 10ms PRP statistically significant differences were observed between $218 \mathrm{kPa}$ and $335 \mathrm{kPa}$ for all harmonic $(1 \mathrm{MHz}, 1.5 \mathrm{MHz}, 2 \mathrm{MHz})$ and the first ultra-harmonic $(1.25 \mathrm{MHz})$ frequencies. The general trend in ICD values within the harmonic frequencies shows an increase at the second harmonic $(1.5 \mathrm{MHz})$ and a decrease at the third harmonic $(2 \mathrm{MHz})$ relative to the first harmonic $(1 \mathrm{MHz})$ at all pressures. Relative to the first ultra-harmonic, the ICD values decrease at each subsequent ultra-harmonic frequencies for both $335 \mathrm{kPa}$ and $908 \mathrm{kPa}$. At $100 \mathrm{~ms}$, statistically significant differences were observed between $218 \mathrm{kPa}$ and $335 \mathrm{kPa}$ at the second and third ultraharmonic frequencies $(1.75 \mathrm{MHz}, 2.25 \mathrm{MHz})$. The general trend in ICD values within both the harmonic $(1 \mathrm{MHz}, 1.5 \mathrm{MHz}, 2 \mathrm{MHz})$ and ultra-harmonic $(1.25 \mathrm{MHz}, 1.75 \mathrm{MHz}$ and $2.25 \mathrm{MHz})$ frequencies follows a similar trend as the 10ms PRP treatment, however the ICD values are significantly lower for the $100 \mathrm{~ms}$ PRP treatment. 

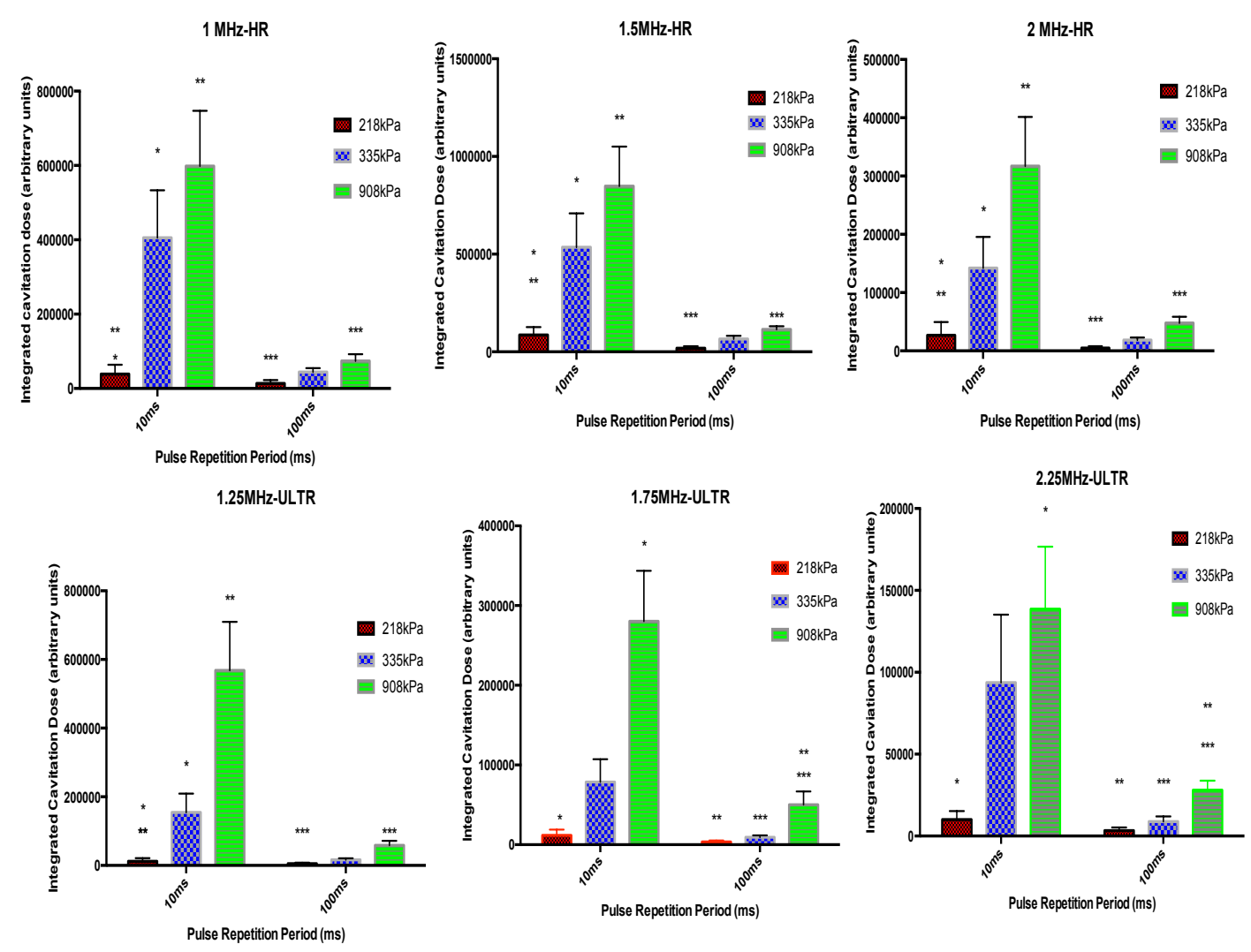

Figure 3.6: Integrated cavitation dose of the harmonic and ultra-harmonic frequency at different acoustic pressures $(218 \mathrm{kPa}, 335 \mathrm{kPa}, 908 \mathrm{kPa})$ received with $2.25 \mathrm{MHz}$ transducer at a PRP of $10 \mathrm{~ms}$ and $100 \mathrm{~ms}$ for $30 \mathrm{~s}$ of treatment time.

\subsection{The relationship between non-viable cells and ICD}

Figure (3.7) shows the correlation of non viable cells and integrated cavitation dose for harmonic and ultra-harmonic exposed to a 30s, 8 cycles $0.5 \mathrm{MHz}$ frequency pulse at different acoustic pressures $(218 \mathrm{kPa}, 335 \mathrm{kPa}, 908 \mathrm{kPa})$ and received with a $2.25 \mathrm{MHz}$ transducer. As the applied pressure was increased, the integrated cavitation dose and non-viable cells also increased. Results did not show any correlation between non-viable cells and ICD. For both PRPs (10ms and $100 \mathrm{~ms})$, exposure of the highest pressure $(908 \mathrm{kPa})$ resulted in the largest non-viable cell values 
$(\sim 22 \%$ and $\sim 26 \%)$ for all harmonic $(1 \mathrm{MHz}, 1.5 \mathrm{MHz}, 2 \mathrm{MHz})$ and ultra-harmonic ( $1.25 \mathrm{MHz}$, $1.75 \mathrm{MHz}, 2.25 \mathrm{MHz})$ frequencies. However, the non-viable cell values obtained from the $10 \mathrm{~ms}$ exposure were higher than that obtained from 100ms. The ICD values for each frequency window measured for both $10 \mathrm{~ms}$ and $100 \mathrm{~ms}$ were not equivalent, each with its own unique specific value. Although there appears to be a relationship between the ICD and cell non-viability, the correlation was not statistically significant. 

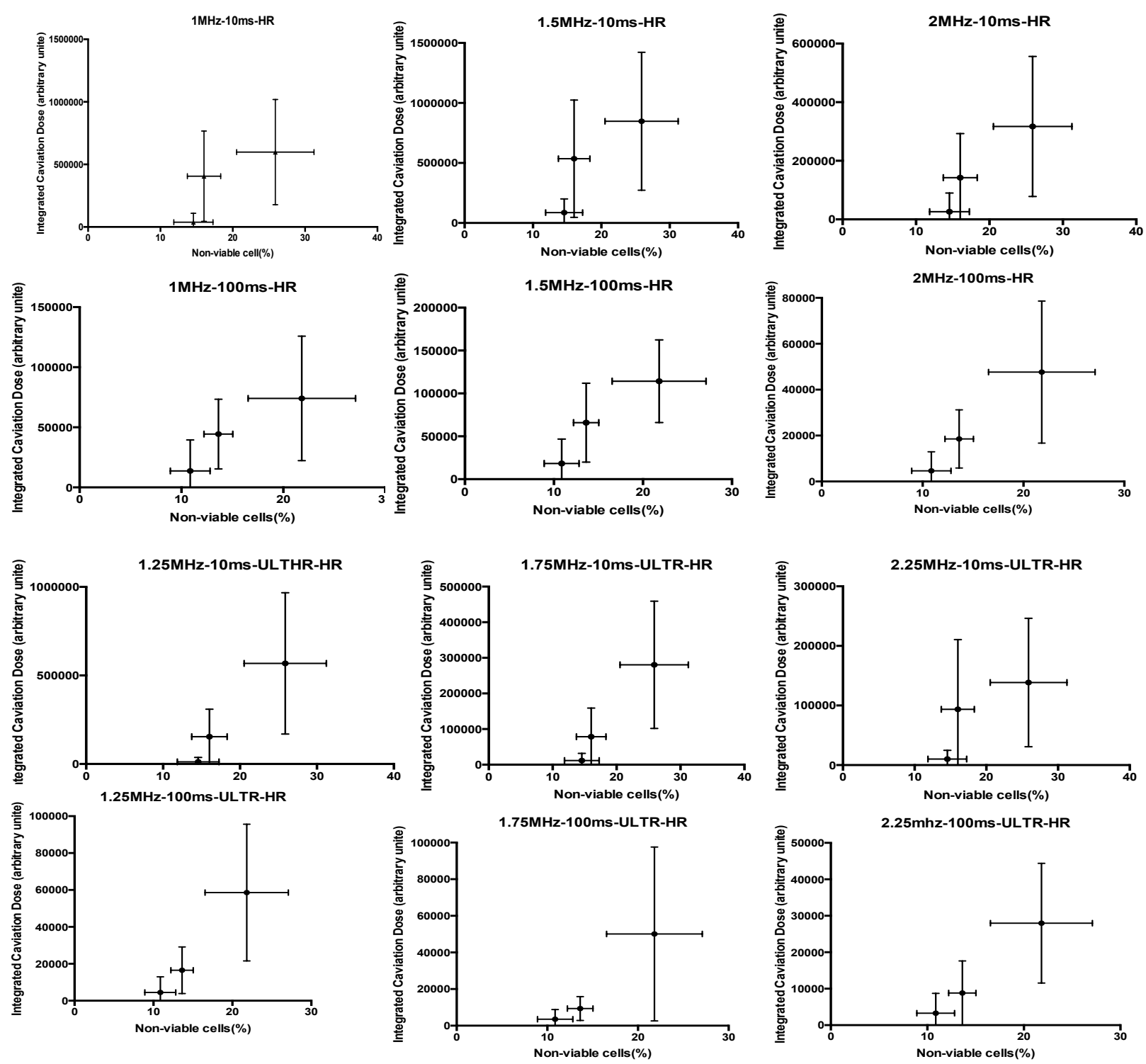

Figure 3.7: The correlation of MDA-MB-231 breast cancer cells vs. integrated cavitation dose of harmonic (2f, 3f, 3f) and ultra-harmonic (2.5f, 3.5f, 4.5f) at different pressure (218kPa, $335 \mathrm{kPa}$,and $908 \mathrm{kPa}$ ), frequencies received with $2.25 \mathrm{MHz}$ transducer exposed to an 8-cycle pulse sequence with a $10 \mathrm{~ms}$ and $100 \mathrm{~ms}$ PRP for a 30 s treatment time $(\mathrm{n}=8)$. 


\section{Chapter 4 Discussion}

\subsection{Introduction}

This study aimed at improving our understanding of the relationship between cells killed by USMB and microbubble acoustic response at different PNP and PRP using passive cavitation detection method and inertial cavitation dose. The study indicates that cell viability and acoustic response of MBs depend on ultrasound pressure and do not vary with PRPs of 10ms and 100ms.

\subsection{Non-viable cells}

It has been shown that ultrasound in the absence of microbubbles at exposure conditions similar to this study induce minimal or no effect on cell viability [36]. In the presence of microbubbles, higher PNPs have a more significant effect on cell death, which may be due to increased damage inflicted on the cell membrane by USMB [33, 39]. In addition, ultrasound and microbubbles has also been shown to cause stresses on the cell membrane increasing the incidence of apoptosis [31]. Whereas low ultrasound pressures have been shown to increase permeabilization of the membrane and allow large molecules to enter the cell and remain viable [41]. The integrated cavitation dose indicates that the MB response increases at higher acoustic pressures. Increasing the PRP from $10 \mathrm{~ms}$ to $100 \mathrm{~ms}$ reduces the number of ultrasound pulses sent to the sample within the treatment time. This may suggest that the increased rate of ultrasound pulses may be causing 
increased levels of damage to the cell membrane or the lengthened gap between pulses may allow the cell additional time to recover from the exposure.

\subsection{Acoustic characteristics of Microbubbles}

The non-linear response of mircobubbles increased with pressure; the amplitude of harmonics and ultra-harmonics signals emitted from the MB increased with pressure. Studies have shown similar results that change in PNP cause significant changes in MB behavior showing irregular oscillations $[24,25,26]$. The spectrogram in (Figure 3.4) present the time dynamics of the MBs acoustic behavior exposed to a pulse with a pulse repetition period of $10 \mathrm{~ms}$ respectively at different pressures. This form of analysis can detect the loss of MBs from the solution as a result of MB disruption. As pressure was increased from $218 \mathrm{kPa}$ to $908 \mathrm{kPa}$, the spectrogram showed subsequently stronger MB signals within 1s and progressively faster loss of MB signals, with the fastest loss at $908 \mathrm{kPa}$ (Figure $3.4 \mathrm{~F}$ ). This suggests that higher PNPs cause stronger non-linear signals, apparent from the harmonic and ultra-harmonic signals, and the disruption of microbubbles at a higher rate. This observation is in accordance with past research papers that highlighted the dependence of MB rupture on PNP [23,24,25]. This information not only provides us with the behavior of microbubbles but also the stability of microbubbles at different pressures.

The integrated cavitation dose (Figures 3.8) at specific frequencies such as harmonic signals (2f, $3 \mathrm{f}, 4 \mathrm{f}$,) and ultra-harmonic signals $(2.5 \mathrm{f}, 3.5 \mathrm{f}, 4.5 \mathrm{f})$, increased with pressure, which has been shown by previously published papers $[43,44,45]$. This may be due to non-linear microbubble oscillations or microbubble disruption. In addition, ICD also increased with PRP which may be due to the number of times MBs within the solution were exposed to US pulses within the $30 \mathrm{~s}$ 
exposure time. At 10ms PRP, MBs are exposed to an US pulse 3000 times, whereas at $100 \mathrm{~ms}$ the MBs are exposed to an US pulse 300 times.

\subsection{Relationship of non-viable cells and integrated cavitation dose}

Generally, as ICD increases the percentage of non-viable cells increases at all harmonic and ultraharmonic frequencies. However, statistically there is no correlation between non-viable cells and ICD. More cells are killed with USMB at higher ICD doses. It is postulated that higher ICD would induce a higher level of stress onto cell membranes [46]. It is also important to note that the proximity of the MB is important for the effect to be realized. The energy emitted from the MB will only do damage to the membrane if it close enough to the surface [47].

\subsection{Limitations of this study}

This study has several limitations. The analysis of passive cavitation detection data did not take into account the bandwidth of the receiving transducer $(2.25 \mathrm{MHz})$. Cell viability was measured using Propidium iodide a few hours after the ultrasound exposure. Propidium iodide is a shortterm analysis that only measures cell death. In addition, only two PRP and three PNP settings were used in this study. 


\section{Chapter 5 Conclusions and Future Work}

\subsection{Conclusions}

The results show that the number of non-viable cells and ICD increases with PNP. The study suggests that there is no correlation between ICD and cell viability. In addition, cell viability appeared to be independent of PRP at the setting used in this study, although the ICD dose increased with PRP. A higher ICD was measured at 10ms PRP compared to $100 \mathrm{~ms}$ PRP. Overall, no correlation was observed between ICD at a specific harmonic or ultra-harmonic frequencies and cell viability.

\subsection{Future Work}

Enhancing our understanding of the acoustic behaviors of MBs and the effect they have on cells is important for improving ultrasound and microbubble therapy. Further focus on the effect of PNP and the corresponding integrated cavitation dose on cell viability is valuable as it will allow for more controlled treatments during experiments. The effect of MB type and size is also important for quantifying MB response. 


\section{Reference}

[1] Prince, J.L., Links, J.M. Medical Imaging Signals and Systems (2006). Upper Saddle River, NJ: Pearson Prentice Hall

[2] Iwanaga, K. K., Tominaga, K. K., Yamamoto, K. K., Habu, M. M., Maeda, H. H., Akifusa, S. S. (2007). Local delivery system of cytotoxic agents to tumors by focused sonoporation. Cancer GeneTherapy, 14(4), 354-363

[3] Karshafian, R., Giles, A., Burns, P., Czarnota, G.J. (2009). Ultrasound-activated Microbubbles as Novel Enhancers of Radiotherapy in Leukemia Cells in Vitro. IEEE International Ultrasonics Symposium Proceedings

[4] Karshafian, R., Samac, S., Bevan, P.D., Burns, P.N(2010). Microbubble mediated sonoporation of cells in suspension: Clonogenic viability and influence of molecular size on uptake. Ultrasonics 50, 691-697

[5] de Jong, N., Emmer, M., van Wamel, A., \& Versluis, M. (2009). Ultrasonic characterization of ultrasound contrast agents. Medical \& Biological Engineering \& Computing, 47(8), 861-873.

[6] Liu, Y., Miyoshi, H., \& Nakamura, M. (2006). Encapsulated ultrasound microbubbles: Therapeutic application in drug/gene delivery. Journal of Controlled Release, 114(1), 89-99.

[7] Wu, J., \& Nyborg, W. L. (2008). Ultrasound, cavitation bubbles and their interaction with cells. Advanced Drug Delivery Reviews, 60(10), 1103-1116.

[8] Cobbold, R.S.C. (2007). Foundations of biomedical ultrasound. Publisher Oxford; Toronto: Oxford University press.

[9] Roy, R. A., Madanshetty, S. I., \& Apfel, R. E. (1990). An acoustic backscattering technique for the detection of transient cavitation produced by microsecond pulses of ultrasound. Journal of the Acoustical Society of America, 87(6), 2451-2458.

[10] Gibbs, V., Cole, D., Sassano, A. (2009). Ultrasound Physics and Technology How, Why and When, Elsevier Limited, China, 45-47p.

[11] Bailey, M.R., Khokholva, V.A., Sapozhnikov, O.A., Kargl, S.G., Crum, L.A. (2002). Physical Mechanisms of the Therapeutic Effect of Ultrasound (A Review).

[12] Ter Haar, G. (2006). Therapeutic applications of ultrasound. Prog Biophys Mol Biol, 93(1-3), 111-29.

[13] Liu, Y., Miyoshi, H., \& Nakamura, M. (2006). Encapsulated ultrasound microbubbles: Therapeutic application in drug/gene delivery. Journal of Controlled Release, 114(1), 89-99. 
[14] O Brien,J., William D.(2007).Ultrasound-biophysics mechanism.Progress in Biophysics and Molecular Biology,93(1-3),212-255.doi:10.1016/pbiomolbio.2006.070010

[15] Ter Haar, G. (1999). Therapeutic ultrasound. European Journal of Ultrasound, 9(1), 3-9.

[16] Baker, K. G., Robertson, V. J., \& Duck, F. A. (2001). A review of therapeutic ultrasound: Biophysical effects. Physical Therapy, 81(7), 1351.

[17] Brady, L.W., Baert, A.L. (1998). Medical radiology: diagnostic imaging and radiation oncology. Berlin: Springer.

[18] Mo, S., Coussios, C., Seymour, L., Carlisle, R. (2012). Ultrasound-enhanced drug delivery for cancer. Expert Opinion on Drug Delivery, 9(12), 1525-1538.

[19] Sboros, V. (2008). Response of contrast agents to ultrasound. Advanced drug delivery reviews, 60(10): 1117-36.

[20] Ghaleb A. Husseini William G. Pitt and Bryant J. Staples (2004). Ultrasonic Drug Delivery A General Review. Expert Opin Drug Delivery, 1(1): 37-56.

[21] Mayer, C. R., \& Bekeredjian, R. (2008). Ultrasonic gene and drug delivery to the cardiovascular system. Advanced Drug Delivery Reviews, 60(10), 1177-1192.

[22] Ferrara, K., Pollard, R., Borden, M. (2007). Ultrasound microbubble contrast agents: fundamentals and application to gene and drug delivery. Annual review of biomedical engineering 9: $415-47$.

[23] Szabo, Thomas L. (2004). Diagnostic Ultrasound Imaging: Inside out. Burlington, MA: Elsevier Academic.

[24] D'Agostino, L., Salvetti, M.V. (2007). Fluid Dynamics of Cavitation and Cavitating Turbopumps. SpringerWien: NY, vol. 496.

[25] Van Bavel, E. (2007). Effects of shear stress on endothelial cells: possible relevance for ultrasound applications. Prog Biophys Mol Biol, 93 (1-3), 374-83

[26] Unger, E.C., Hersh, E., Vannan, M., Matsunaga, T.O., McCreery, T. (2001). Local drug and gene delivery through microbubbles. Prog. Cardiovasc. Dis. 44, 45-54.

[27] Chen, W., Matula, T. J., Brayman, A. A., \& Crum, L. A. (2003). A comparison of the fragmentation thresholds and inertial cavitation doses of different ultrasound contrast agents. The Journal of the Acoustical Society of America, 113(1), 643. 
[28] H. Tang, C.C.J. Wang, D. Blankschtein, and R. Langer. An investigation of the role of cavitation in low-frequency ultrasound-mediated transdermal drug transport Pharmaceutical Research, 19(8):1160-1169, 2002.

[29] Torpy, J. M., Lynm, C. (2010). Cancer: The basics. Jama, 304(14), 1628-1628.

[30] Forbes, M. M., Steinberg, R. L., \& O'Brien Jr., W. D. (2008). Examination of inertial cavitation of optison in producing sonoporation of chinese hamster ovary cells. Ultrasound in Medicine and Biology, 34(12), 2009-2018.

[31] Guzmán, H. R., Nguyen, D. X., Khan, S., \& Prausnitz, M. R. (2001). Ultrasoundmediated disruption of cell membranes. I. quantification of molecular uptake and cell viability. The Journal of the Acoustical Society of America, 110(1), 588.

[32] Meijering, Bernadet D. M., Robert H. Henning, Wiek H. Van Gilst, Ivana Gavrilović, Annemieke Van Wamel, and Leo E. Deelman. (2007) "Optimization of Ultrasound and Microbubbles Targeted Gene Delivery to Cultured Primary Endothelial Cells." Journal of Drug Targeting 15.10: 664-71.

[33] Karshafian, Raffi, Peter D. Bevan, Ross Williams, Sanya Samac, and Peter N. Burns.(2009)."Sonoporation by Ultrasound-Activated Microbubble Contrast Agents: Effect of Acoustic Exposure Parameters on Cell Membrane Permeability and Cell Viability." Ultrasound in Medicine \& Biology 35.5: 847-60.

[34] Madanshetty, S.I., Roy, R.A., Apfel, R.E. (1991). Acoustic microcavitation: its active and passive acoustic detection. The Journal of the Acoustical Society of America, 90(3): 1515-26.

[35] King, D.A., Santin, M. Malloy, M.J., Roberts, A.C., Haak, A., Foiret, J., Haupert, S., Jafari, S., Bridal, L., O'Brien, W.D. (2009). Using passive cavitation detection to observe postexcitation response of ultrasound contrast agents. IEEE International Ultrasonics Symposium, pages $1286-1289$.

[36] Chun-Yen Lai, Chia-Hsuan Wu, Chia-Chun Chen, and Pai-Chi Li. (2006). Quantitative relations of acoustic inertial cavitation with sonoporation and cell viability. Ultrasound in medicine \& biology, 32(12): 1931-41.

[37] Qiu, Y., Luo, Y., Zhang, Y., Cui, W., Zhang, D., Wu, J., Zhang, J., Tu, J. (2010). The correlation between acoustic cavitation and sonoporation involved in ultrasound-mediated DNA transfection with polyethylenimine (PEI) in vitro. Journal of controlled release: o cial journal of the Controlled Release Society, 145(1):40-8.

[38] Ammi, A. Y., Cleveland, R. O., Mamou, J., Wang, G. I., Bridal, S. L., \& O'Brien Jr., W. D. (2006). Ultrasonic contrast agent shell rupture detected by inertial cavitation and rebound signals. IEEE Transactions on Ultrasonics, Ferroelectrics, and Frequency Control, 53(1), 126135. 
[39] Miller, D. L., Pislaru, S. V., \& Greenleaf, J. E. (2002). Sonoporation: Mechanical DNA delivery by ultrasonic cavitation. Somatic Cell and Molecular Genetics, 27(1-6), 115-134. doi:10.1023/A:1022983907223

[40] Nikolova-Karakasjian MN, Rozenova K (2010) Sphingolipids as Signaling and Regulatory Molecules, eds C Chalfant and M Del Poeta (Landes Bioscience and Springer Science and Business Media, New York), pp 86-107.

[41] Bao, S., Thrall, B. D., \& Miller, D. L. (1997). Transfection of a reporter plasmid into cultured cells by sonoporation in vitro.Ultrasound in Medicine \& Biology, 23(6), 953-959. doi:10.1016/S0301-5629(97)00025-2

[42] Qin, S., Caskey, C. F., \& Ferrara, K. W. (2009). Ultrasound contrast microbubbles in imaging and therapy: Physical principles and engineering. Physics in Medicine and Biology,54(6), R27-R57. doi:10.1088/0031-9155/54/6/R01

[43] Chun-Yen Lai,Chia-Hsuan, Chia-Chun Chen, and Pai-Chi Li(2006). Quantitative Relation of acoustic inertial cavitation with sonoporation and cell viability. Ultrasound in medicine \& biology, 32(12):1931-41

[44] Yuanyuan Qiu, Yi Luo, Yanli Zhang, Weicheng Cui, Dong Zhang, Junru Wu, Jun-feng Zhang, and Juan Tu. (2010). The correlation between acoustic cavitation and sonoporation involved in ultrasound-mediated DNA transfection with polyethylenimine (PEI) in vitro. Journal of controlled release, journal of the Controlled Release Society, 145(1):40-8.

[45] Wen-Shiang Chen, Andrew A. Brayman, Thomas J. Matula, and Lawrence A. Crum. (2003). Inertial cavitation dose and hemolysis produced in vitro with or without Optison. Ultrasound in Medicine \& Biology, 29(5):725-737.

[46] Hwang, J. H., Brayman, A. A., Reidy, M. A., Matula, T. J., Kimmey, M. B., \& Crum, L. A. (2005). Vascular effects induced by combined 1-MHz ultrasound and microbubble contrast agent treatments in vivo. Ultrasound in Medicine \& Biology,31(4), 553-564. doi:10.1016/j.ultrasmedbio.2004.12.014

[47] Ahmet Tezel and Samir Mitragotri.(2003). Interactions of inertial cavitation bubbles with stratum corneum lipid bilayers during low-frequency sonophoresis (2003). Biophysical journal, 85(6):3502-12. 